NUREG/CR-6035

\title{
Feasibility Study for Improved \\ Steady-State Initialization \\ Algorithms for the \\ RELAP5 Computer Code
}

Phase I Improved Steady-State Initialization

Algorithms for Computer Codes

Prepared by

M. P. Paulsen, C. E. Peterson, K. R. Katsma

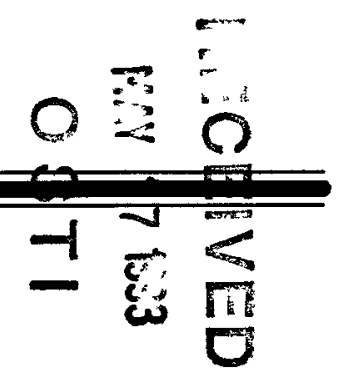

Computer Simulation \& Analysis, Inc.

Prepared for

U.S. Nuclear Regulatory Commission 


\section{DISCLAIMER}

This report was prepared as an account of work sponsored by an agency of the United States Government. Neither the United States Government nor any agency Thereof, nor any of their employees, makes any warranty, express or implied, or assumes any legal liability or responsibility for the accuracy, completeness, or usefulness of any information, apparatus, product, or process disclosed, or represents that its use would not infringe privately owned rights. Reference herein to any specific commercial product, process, or service by trade name, trademark, manufacturer, or otherwise does not necessarily constitute or imply its endorsement, recommendation, or favoring by the United States Government or any agency thereof. The views and opinions of authors expressed herein do not necessarily state or reflect those of the United States Government or any agency thereof. 


\section{DISCLAIMER}

Portions of this document may be illegible in electronic image products. Images are produced from the best available original document. 


\section{AVAILABILITY NOTICE}

Availability of Reference Materials Cited in NRC Publications

Most documents clted in NRC publications will be avallable from one of the following sources:

1. The NRC Public Document Room. 2120 L Street. NW., Lower Level, Washington, DC 20555

2. The Superintendent of Documents, U.S. Government Printing Office, P.O. Box 37082, Washington, DC 20013-7082

3. The National Technical Information Service, Springfield, VA 22161

Although the listing that follows represents the majority of documents cited in NRC publications, it is not intended to be exhaustive.

Referenced documents avallable for inspection and copying for a fee from the NRC Public Document Room include NRC correspondence and internal NRC memoranda; NRC bulletins, circulars, information notices, Inspection and investigation notices; licensee event reports; vendor reports and correspondence; Commission papers; and applicant and licensee documents and correspondence.

The following documents in the NUREG series are available for purchase from the GPO Sales Program: formal NRC staff and contractor reports, NRC-sponsored conference proceedings, international agreement reports, grant publications, and NRC booklets and brochures. Also available are regulatory guides, NRC regulations in the Code of Federal Regulations, and Nuclear Regulatory Commission Issuances.

Documents available from the National Technical Information Service include NUREG-series reports and technical reports prepared by other Federal agencies and reports prepared by the Atomic Energy Commission, forerunner agency to the Nuclear Regulatory Commission.

Documents available from public and special technical libraries include all open literature items, such as books, Journal articles, and transactions. Federal Register notices. Federal and State legislation, and congressional reports can usually be obtained from these libraries.

Documents such as theses, dissertations, foreign reports and translations, and non-NRC conference proceedings are avallable for purchase from the organization sponsoring the publication cited.

Single coples of NRC draft reports are available free, to the extent of supply, upon written request to the Office of Administration, Distribution and Mail Services Section, U.S. Nuclear Regulatory Commission, Washington. DC 20555.

Copies of industry codes and standards used in a substantive manner in the NRC regulatory process are maintained at the NRC Library, 7920 Norfolk Avenue, Bethesda, Maryland, for use by the public. Codes and standards are usually copyrighted and may be purchased from the originating organization or, if they are American National Standards, from the American National Standards Institute, 1430 Broadway, New York, NY 10018.

\section{DISCLAIMER NOTICE}

This report was prepared as an account of work sponsored by an agency of the United States Government. Neither the United States Government nor any agency thereof, or any of their employees, makes any warranty, expressed or implied, or assumes any legal liability of responsibility for any third party's use, or the results of such use, of any information, apparatus, product or process disclosed in this report, or represents that its use by such third party would not infringe privately owned rights. 


\section{Feasibility Study for Improved Steady-State Initialization Algorithms for the RELAP5 Computer Code}

Manuscript Completed: March 1993

Date Published: April 1993

Prepared by

M. P. Paulsen, C. E. Peterson, K. R. Katsma

Computer Simulation \& Analysis, Inc.

P. O. Box 51596

Idaho Falls, ID 83405

Prepared for

Division of Systems Research

Office of Nuclear Regulatory Research

U.S. Nuclear Regulatory Commission

Washington, DC 20555

NRC FIN L 2565 



\begin{abstract}
A design for a new steady-state initialization method is presented that represents an improvement over the current method used in RELAP5. Current initialization methods for RELAP5 solve the transient fluid flow balance equations simulating a transient to achieve steady-state conditions. Because the transient solution is used, the initial conditions may change from the desired values requiring the use of controllers and long transient running times to obtain steady-state conditions for system problems. The new initialization method allows the user to fix thermal-hydraulic values in volumes and junctions where the conditions are best known and have the code compute the initial conditions in other areas of the system. The steady-state balance equations and solution methods are presented. The constitutive, component, and special purpose models are reviewed with respect to modifications required for the new steady-state initialization method. The requirements for user input are defined and the feasibility of the method is demonstrated with a testbed code by initializing some simple channel problems. The initialization of the sample problems using the old and the new methods are compared.
\end{abstract}





\section{CONTENTS}

Section

Page

1 SUMMARY

2 STEADY-STATE INITIALIZATION

3 HYDRODYNAMIC MODEL

3.1 Steady-State Field Equations $\quad 5$

3.2 Initial Condition Specification $\quad 8$

3.2.1 Volume Pressure and Junction Loss Coefficients 9

$\begin{array}{lr}3.2 .2 \text { Fluid Internal Energy } & 9\end{array}$

3.2.3 Junction Flow Rates 10

3.2.4 Vapor Void Fraction 10

3.2.5 Initial Volume State Properties With Noncondensibles 10

$\begin{array}{ll}3.2 .6 \text { Required User Input } & 10\end{array}$

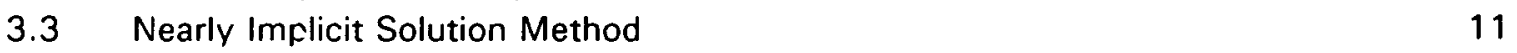

$\begin{array}{ll}3.4 & \text { Equation-of-State Relationships }\end{array}$

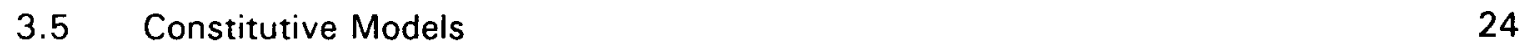

3.5.1 Flow Regime Maps $\quad 25$

$\begin{array}{ll}3.5 .2 \text { Interphase Friction } & 25\end{array}$

3.5.3 Coefficient of Virtual Mass $\quad 25$

$\begin{array}{ll}3.5 .4 \text { Wall Friction } & 25\end{array}$

3.5.5 Wall Heat Transfer 26

3.5.6 Interphase Heat Transfer 26

3.6 Special Process Models $\quad 26$

3.6.1 Choked Flow 26

$\begin{array}{ll}3.6 .2 \text { Horizontal Stratification and Entrainment } & 27\end{array}$

$\begin{array}{ll}3.6 .3 \text { Abrupt Area Change } & 27\end{array}$

3.6.4 Crossflow Junction 27

$\begin{array}{ll}3.6 .5 \text { Water Packing Mitigation } & 27\end{array}$

3.6.6 Counter Current Flow Limitation $\quad 27$

$\begin{array}{lll}3.7 & \text { Component Models } & 27\end{array}$

$\begin{array}{ll}3.7 .1 \text { Branch } & 28\end{array}$

3.7.2 Separator 28

$\begin{array}{ll}3.7 .3 \text { Jet Mixer } & 28\end{array}$

$\begin{array}{ll}\text { 3.7.4 Centrifugal Pump Model } & 29\end{array}$

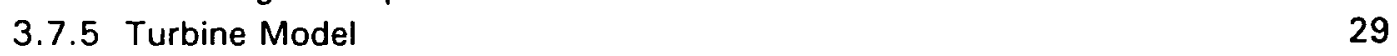

$\begin{array}{ll}3.7 .6 \text { Valves } & 29\end{array}$

3.7.7 Accumulator 30

3.7.8 ECC Mixer Model $\quad 30$ 


\section{CONTENTS}

Section

$4 \quad$ HEAT STRUCTURE

4.1 Conduction Equation and Finite Differencing - Transient Form 33

4.2 Steady-State Procedure

5 CONTROL SYSTEM

6 TRIP SYSTEM

7 REACTOR KINETICS

8 OVERALL SYSTEM ENERGY BALANCE

8.1 Flow Network Identification 43

$\begin{array}{lll}8.2 & \text { PWR System Energy Balance } & 43\end{array}$

8.2.1 Steam Generators $\quad 44$

$9 \quad$ IMPLEMENTATION PLAN $\quad 47$

$\begin{array}{lll}9.1 & \text { Flow Diagrams } & 47\end{array}$

9.1.1 Input - Initialization Modifications $\quad 47$

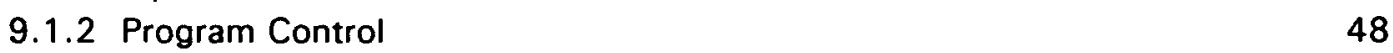

$\begin{array}{ll}9.1 .3 \text { Iteration Control } & 48\end{array}$

$\begin{array}{lll}9.2 & \text { Input Modifications } & 48\end{array}$

9.2.1 Initial Volume Thermal-Hydraulic Conditions $\quad 52$

9.2.2 Junction Loss Coefficients and Flow $\quad 52$

9.2.3 Overall Energy Balance Specification $\quad 53$

9.2.4 Steady-State Convergence Criteria $\quad 53$

$\begin{array}{lll}9.3 & \text { Maintaining Code Modifications } & 53\end{array}$

10 DEMONSTRATION ANALYSIS $\quad 55$

10.1 Test Problem 1 $\quad 55$

10.2 Test Problems 2, 3, and $4 \quad 58$

$\begin{array}{lll}10.3 & \text { Test Problem } 5 & 58\end{array}$

11 REFERENCES $\quad 63$ 


\section{LIST OF FIGURES}

Figure $\quad$ Page

4.1 RELAP5 conductor solution procedure $\quad 32$

4.2 Comparison of RELAP5 transient and steady-state conduction subroutines 36

9.1 Partial flow diagram for steady-state read and initialization 49

$\begin{array}{lll}9.2 & \text { Partial diagram for program control } & 50\end{array}$

9.3 Transient and steady-state advancement schemes

10.1 Noding diagram for FRIGG test FT-6

10.2 Schematic diagrams for test problems 2, 3, and $4 \quad 60$ 


\section{LIST OF TABLES}

Table

Page

10.1 Pressure and loss coefficient convergence steady-state test problem

10.2 RELAP5 pressure and velocity solution

59

10.3 Data for test problems 2, 3, and 4

61

10.4 Calculation results for problems 2,3 , and 4

62 


\section{ABBREVIATIONS}

$\begin{array}{ll}\text { BWR } & \text { Boiling Water Reactors } \\ \text { CHF } & \text { Critical Heat Flux } \\ \text { CPU } & \text { Central Processor Unit } \\ \text { ECC } & \text { Emergency Core Cooling } \\ \text { ECCS } & \text { Emergency Core Cooling System } \\ \text { HTC } & \text { Heat Transfer Correlation } \\ \text { LHS } & \text { Left-Hand Side } \\ \text { LWR } & \text { Light Water Reactor } \\ \text { PWR } & \text { Pressurized Water Reactor } \\ \text { RCP } & \text { Reactor Coolant Pump } \\ \text { RCS } & \text { Reactor Coolant System } \\ \text { RHS } & \text { Right-Hand Side } \\ \text { SBIR } & \text { Small Business Innovative Research }\end{array}$




\section{SUMMARY}

Many LWR operational transient simulations are initiated from steady-state conditions by small perturbations. It is important that these simulations begin from a true steady state so that deviations from a steady-state condition do not mask the true transient behavior. RELAP5 currently solves the transient fluid flow balance equations simulating a transient until a steady-state solution can be obtained (Ref. 1). This requires relatively long transients to be run to obtain steady-state conditions, after which the user must restart the problem at the end of the "null" transient. Revised input must then be supplied at restart to initiate the transient.

Using a transient solution to obtain a steady state has the potential for introducing problems. The transient solutions are initial value problems and require that the initial mass and energy inventories be supplied by the code user, typically using pressure, temperature, and quality. The transient solution then rearranges the initial mass and energy distribution to one that satisfies some steadystate condition. This also changes the pressure, temperature, quality and flow distributions and the final steady state may not be the desired steady state. To alleviate this problem, control systems and/or special purpose coding is used to adjust boundary conditions to force the desired steady state, e.g., mass flow rates, mass and energy inventories, and liquid levels. While this is a legitimate approach, it makes it difficult to use the code and increases the experience required to become a competent RELAP5 user.

A true steady-state solution method has been designed for RELAP5 and implemented on a limited basis in a testbed code to demonstrate the feasibility of the method. Several single-phase demonstration problems run with the testbed code demonstrated that a steady state could be obtained in five iterations or less (eight significant figure accuracy). Each iteration is computationally equivalent to a time-step advancement. The same sample problems were run with the standard RELAP5 initialization method. These results agreed with the testbed code yet, required between 8 and 108 time steps to reach the same convergence even when the correct flow was input.

The results obtained from the testbed code demonstration calculations indicate that steady-state condition can be obtained rapidly. Based on the testbed code experience, it is probable that a large system model could be initialized in 15 to 40 iterations. This has the potential of saving computer time and more importantly improving the productivity of the analysts. This steady-state initialization will allow the user to initialize the model and perform the transient calculation in a single run rather than running a null transient to obtain initial conditions, and then restarting the problem to supply transient-specific input.

The design for steady-state initialization is such that it is a separate module that will have minimum impact on the existing coding and the user. Many of the subroutines used in the steady-state solution will be existing RELAP5 subroutines that will not require modification. Subroutines that will have to be modified will require only minor changes. The design presented here not only minimizes the impact on incorporating the steady-state coding, but also incorporates the steady-state solution 
as an option which will minimize any input changes. The user will be able to run RELAP5 with the current input models and current options and only a small amount of additional input will be required if the steady-state initialization option is chosen.

The work documented herein represents the completion of Phase I of a SBIR project to examine improved steady-state initialization algorithms for computer codes. In particular, this work has focused on the RELAP5 code. The steady-state field equations and the solution method are presented. The constitutive, component and special process models are reviewed with respect to any special treatment that may be required for steady-state initialization. The treatment of how other models (such as control systems, trip system, and reactor kinetics will be treated during steady-state initialization is also discussed. Finally, the implementation plan and the results of the demonstration analyses are presented. 


\section{STEADY-STATE INITIALIZATION}

The problems associated with using the transient solution to obtain initial conditions can be resolved by incorporating a solution to the steady-state form of the governing fluid flow and heat transfer balance equations. Using the steady-state form of the equations can produce accurate steady-state conditions in a fraction of the computer time and manpower that the current methods require. The user can specify initial conditions for the steady-state equations in locations where the parameters such as pressure, temperature, and flow are known best. These specified initial conditions will not be changed during the steady-state solution and the user will not be required to use the control system to try to maintain the known initial conditions as is done with the current method.

A study was undertaken to determine the feasibility of implementing a steady-state solution within the framework of the RELAP5 code. The results of the study and demonstration of feasibility are reported herein.

The technical objectives that were accomplished during the study are summarized below.

- The thermal-hydraulic balance equations were cast in steady-state form, the dependent variables determined, and the nearly implicit numerical solution was reviewed and modifications proposed for solving the steady-state equations.

- The heat conduction equation solution, including the appropriate HTC, were reviewed. The steady-state solution was implemented in the testbed code and used in a demonstration problem.

- The power generation models were reviewed and required modifications identified.

- All constitutive and component models were reviewed and steady-state specific modifications designed where necessary.

- The steady-state driver or control program was designed along with the coding needed to define the phasic thermodynamic conditions from the steady-state dependent variables.

- The energy addition to a reactor system is a direct result of the power generation boundary conditions and component models such as electrical heaters and centrifugal pumps. In order to ensure that there is an overall energy balance across the system, a methodology was designed to ensure that an energy balance occurs.

- Several demonstration problems were initialized using the steady-state testbed version of RELAP5 MOD3 (version 80). These initializations were compared to initializations obtained using the standard method. Excellent agreement was obtained between the methods, demonstrating the feasibility of developing a steady-state solution that is consistent with the transient solution. 



\section{HYDRODYNAMIC MODEL}

The steady-state balance equations and the method used to solve these equations are presented in this section. A review of the equation of state, the constitutive models, the component models and the special process models was done to determine what modifications will be required (if any) to incorporate these models in a steady-state solution. All of these models are discussed with respect to steady-state initialization in this section.

The RELAP5 hydrodynamic model is a transient, one-dimensional, two-fluid model for flow of a twophase steam-water mixture that can contain noncondensible gases in the steam phase and a nonvolatile component in the liquid water phase. Two-flund equations of motion form the basis of the hydrodynamic model. Carlson, et al., present the details of the model (Ref. 1).

The RELAP5 hydrodynamic model is used as the basis of the steady-state initialization feature. Two slightly different hydrodynamic models are given in Reference 1, for the semi-implicit solution method and another for the nearly implicit method. An artificial viscosity term is included in the model used for the nearly implicit solution, but it is not included for model used for the nearly implicit method. The work presented herein deals with the nearly implicit form of the model and does not include the artificial viscosity term. This form is consistent with the model used for the nearly implicit transient solution method and will ensure consistency between the steady-state and transient solutions. A truly consistent approach will allow the transient equations to be advanced in time from the initial conditions without changing the initial conditions, provided no forcing functions are imposed on the system. This is referred to as a null transient and is the measure of a truly consistent steady-state solution.

The artificial viscosity model formulation can be considered at a later date and will be necessary to ensure that a null transient can be maintained when the semi-implicit transient solution method is used. Only slight modifications of the steady-state solution will be required and they can be selected based on the transient solution option specified in the input model.

The following sections present the steady-state field equations that are used in the steady-state solution, the initial condition specifications, the nearly implicit solution method that has been adapted for use in the steady-state solution, and the simplifications used to implement the test bed code steady-state solution procedure.

\subsection{Steady-State Field Equations}

The steady-state field equations provide the basis for the steady-state solution. They are obtained from the time-dependent field equations by dropping the time derivative components. When noncondensible gases are considered, seven governing field equations are used. They are comprised of two phasic continuity equations, two phasic energy equations, two phasic momentum equations, and a noncondensible gas continuity equation. The primary dependent variables are the pressure $(P)$, the phasic specific internal energies $\left(U_{g}, U_{f}\right)$, vapor void fraction $\left(\alpha_{o}\right)$, phasic velocities $\left(v_{0}, v_{f}\right)$, and the noncondensible quality $\left(X_{n}\right)$. The independent variable for the one-dimensional spacial coordinate is given by $x$. 
The steady-state vapor continuity equation is

$\frac{1}{A} \frac{\partial}{\partial x}\left[\alpha_{o} \rho_{a} v_{a} A\right]-\Gamma_{0}+\Gamma_{n c}$

and the steady-state liquid continuity equation is

$\frac{1}{A} \frac{\partial}{\partial x}\left[\alpha, \rho, v_{f} A\right]--\Gamma_{0}+\Gamma_{0}$

where

$\begin{array}{lll}A & = & \text { flow area, } \mathrm{m}^{2} \\ x & = & \text { spatial coordinate, } \mathrm{m} \\ \alpha_{0} & = & \text { gas void fraction } \\ \rho_{0} & = & \text { gas density, } \mathrm{kg} / \mathrm{m}^{3} \\ v_{0} & = & \text { gas velocity, } \mathrm{m} / \mathrm{s} \\ \Gamma_{0} & = & \text { vapor generation rate, } \mathrm{kg} / \mathrm{m}^{3}-\mathrm{s} \\ \Gamma_{n c} & = & \text { noncondensible generation rate, } \mathrm{kg} / \mathrm{m}^{3}-\mathrm{s} \\ \alpha_{f} & = & \text { fluid void fraction, } 1-\alpha_{0} \\ \rho_{f} & = & \text { fluid density, } \mathrm{kg} / \mathrm{m}^{3} \\ v_{f} & = & \text { fluid velocity, } \mathrm{m} / \mathrm{s} \\ \Gamma_{s} & = & \text { solute generation rate, } \mathrm{kg} / \mathrm{m}^{3}-\mathrm{s}\end{array}$

The steady-state vapor energy equation is

$$
\begin{aligned}
\frac{1}{A} \frac{\partial}{\partial x}\left[\alpha_{0} \rho_{0} U_{0} v_{0} A\right] & +\frac{P}{A} \frac{\partial}{\partial x}\left[\alpha_{0} v_{0} A\right]=-\left[\frac{h_{f}^{*}}{h_{0}^{*}-h_{f}^{*}}\right] H_{10}\left(T_{s}-T_{0}\right)-\left[\frac{h_{0}^{*}}{h_{g}^{*}-h_{f}^{*}}\right] H_{1 f}\left(T_{s}-T_{f}\right) \\
& +\left[\left(\frac{1+\varepsilon}{2}\right) h_{0 s}+\left(\frac{1-\varepsilon}{2}\right) h_{f o}\right] \Gamma_{w}+Q_{w o}+\text { Diss }_{0}+\Gamma_{n c} H_{n c}
\end{aligned}
$$

and the steady-state liquid energy equation is

$$
\begin{aligned}
& \frac{1}{A} \frac{\partial}{\partial x}\left[\alpha_{f} \rho_{f} U_{f} v_{f} A\right]+\frac{P}{A} \frac{\partial}{\partial x}\left[\alpha_{f} v_{f} A\right]-\left[\frac{h_{f}^{*}}{h_{0}^{*}-h_{f}^{*}}\right] H_{10}\left(T_{0}-T_{0}\right)+\left[\frac{h_{a}^{*}}{h_{0}^{*}-h_{f}^{*}}\right] H_{1}\left(T_{0}-T_{f}\right) \\
& -\left[\left(\frac{1+\epsilon}{2}\right) h_{g s}+\left(\frac{1-\epsilon}{2}\right) h_{f s}\right] \Gamma_{w}+Q_{w f}+\text { Diss }_{f}+\Gamma_{s} H_{s}
\end{aligned}
$$


where

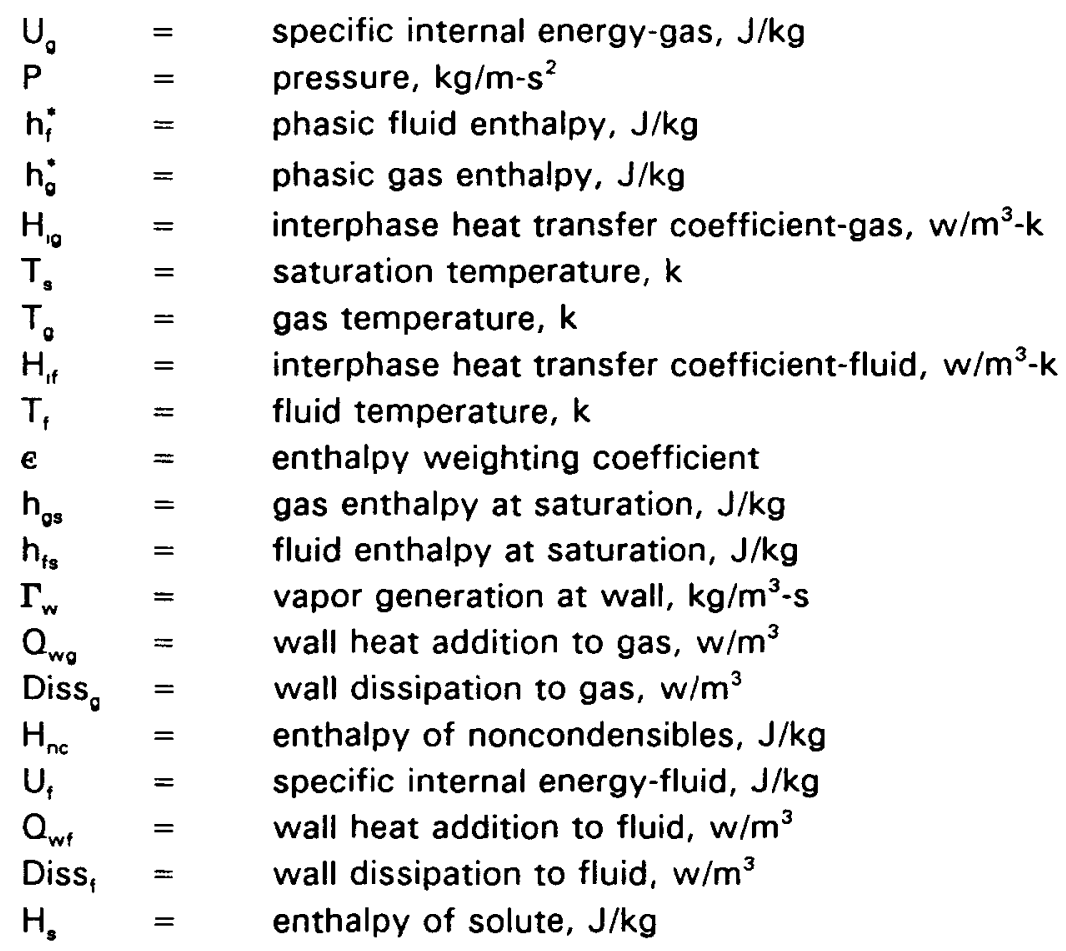

The enthalpy weighting coefficient is defined as follows

$$
\begin{aligned}
& \epsilon-1, \Gamma_{w}>0 \\
& \epsilon-1, \Gamma_{w}<0
\end{aligned}
$$

and the vapor and liquid dissipation are respectively given by

$$
\operatorname{Diss}_{0}-\alpha_{0} \rho_{0} f_{w o} v_{0}^{2}
$$

Diss, $_{f}=\alpha_{f} \rho_{f} f_{w f} v_{f}^{2}$

where

$$
\begin{array}{ll}
f_{w o} & =\text { wall friction factor-gas, } s^{-1} \\
f_{w f} & =\quad \text { wall friction factor-fluid, } s^{-1}
\end{array}
$$

Reference 1 contains the details associated with the mass and energy transfer source terms.

The conditions related to the mass transfer terms are given in the RELAP5 manual. Shown here are the final form of the interphase and wall vapor generation terms. 
The form of the momentum equations used by RELAP5 depends on the solution option selected. An artificial viscosity term is included in the model used with the semi-implicit solution. It is included to improve the method's numerical stability. The nearly implicit solution method (see Section 3.2 ) is used for the steady-state solution and the artificial viscosity terms are not included in the hydrodymanic model. This is consistent with the model used for the nearly implicit transient solution.

The steady-state vapor momentum equation is

$$
\begin{aligned}
& \frac{1}{2} \alpha_{0} \rho_{0} A \frac{\partial v_{0}^{2}}{\partial x}=-\alpha_{0} A \frac{\partial P}{\partial x}+\alpha_{\theta} \rho_{0} B_{x} A-\alpha_{0} \rho_{0} A f_{w o} v_{0}+\Gamma_{\theta} A\left(v_{1}-v_{0}\right) \\
& -\alpha_{0} \rho_{0} A f_{10}\left(C_{1} v_{0}-C_{0} v_{f}\right)-\alpha_{0} \rho_{g} A H_{b} v_{0} \\
& -\Gamma_{n c} v_{g} A+f_{x} \alpha_{f} \alpha_{0} \rho_{0} f_{w_{g}} A v_{0}-f_{x} \alpha_{g} \alpha_{f} \rho_{f} f_{w f} A v_{f}
\end{aligned}
$$

and the steady-state liquid momentum equation is

$$
\begin{aligned}
\frac{1}{2} \alpha_{f} \rho_{f} A \frac{\partial v_{f}^{2}}{\partial x}= & -\alpha_{f} A \frac{\partial P}{\partial x}+\alpha_{f} \rho_{f} B_{x} A-\alpha_{f} \rho_{f} A f_{w f} v_{f}-\Gamma_{g} A\left(v_{t}-v_{f}\right) \\
& -\alpha_{f} \rho_{f} A f_{i f}\left(C_{o} v_{f}-C_{f} v_{g}\right)-\alpha_{f} \rho_{f} A H_{f f} v_{f}-\Gamma_{s} v_{f} A+f_{x} \alpha_{g} \alpha_{f} \rho_{f} f_{w f} A v_{f}-f_{x} \alpha_{f} \alpha_{g} \rho_{g} f_{w g} A v_{g}
\end{aligned}
$$

where

$$
\begin{array}{lll}
B_{x} & = & \text { body forces, } \mathrm{m} / \mathrm{s}^{2} \\
v_{1} & = & \text { interfacial velocity, } \mathrm{m} / \mathrm{s} \\
f_{10} & = & \text { interphase friction, } \mathrm{s}^{-1} \\
\mathrm{C}_{1} & = & \text { drift flux parameter } \\
\mathrm{C}_{0} & = & \text { drift flux profile parameter } \\
\mathrm{H}_{\mathrm{w}} & = & \text { gas loss coefficient, } \mathrm{s}^{-1} \\
\mathrm{f}_{\mathrm{x}} & = & \text { wall friction coefficient } \\
\mathrm{H}_{\mathrm{w}} & = & \text { liquid loss coefficient, } \mathrm{s}^{-1}
\end{array}
$$

As defined below, the interphase velocity is defined to be equal to the appropriate phase velocity.

$$
\begin{aligned}
& v_{1}=v_{f} \text { for } \Gamma_{0}>0 \\
& v_{1}=v_{0} \text { for } \Gamma_{0}<0
\end{aligned}
$$

Finally, the steady-state form of the noncondensible continuity equation is

$$
\frac{1}{A} \frac{\partial}{\partial x}\left[\alpha_{0} \rho_{0} X_{n c} v_{0} A\right]=\Gamma_{n c}
$$


where

$\mathrm{X}_{\mathrm{nc}}=$ noncondensible quality

\subsection{Initial Condition Specification}

The steady-state field equations are solved using appropriate initial conditions to obtain the steady initial state. In order to achieve a steady-state balance, one dependent variable must be left to balance each of the steady-state field equations. Therefore, the user cannot completely specify all the volume state properties and system flow distribution as currently required now. In fact, in the large majority of instances, the user does not know or finds it difficult to accurately compute and supply all that initial information. The proposed steady-state initialization method will allow the user to pick the type and location of thermal-hydraulic input that will remain fixed during steady-state initialization. The user can then fix the information that is best known and let the code compute the remaining information. To be more specific, currently the user must supply the volume fluid state conditions in each fluid volume, specify all junction local loss coefficients and input all junction flow rates. With the new steady-state initialization method, this will not be necessary.

For steady-state initialization, only a minimum set of thermodynamic input will be required along with junction flow rates and local loss coefficients. Therefore, the unknown values for volume pressure, phasic internal energies, junction flow rate and junction loss coefficients will have to be obtained by solving the appropriate steady-state balance equations. The following subsections discuss how the parameters will be obtained and general input requirements for the initial condition specification.

\subsubsection{Volume Pressure and Junction Loss Coefficients}

The steady-state sum momentum balance (Eq. 3-11) can be satisfied by computing volume pressure (either upstream or downstream) or junction form loss coefficients. The user (through input data) will select the junctions where loss coefficients are to be computed, and which input volume pressures are to be maintained. Based on the user input, the steady-state logic will determine whether the sum momentum equations will solve for the unknown junction loss coefficients, or unknown upstream or downstream volume pressure.

\subsubsection{Fluid Internal Energy}

The steady-state phasic energy equations (Eqs. 3-33 and 3-35) will be used to solve for the liquid and gas internal energy. The user will specify the internal energy in any single-phase volume within a flow network and the phasic energy equations will be used to compute the fluid internal energy distribution in the fluid volumes around the rest of the flow network. 


\subsubsection{Junction Flow Rates}

The sum continuity equation will be used to ensure an overall mass flow rate balance exists in the system. Currently the user must input the mass flow rates throughout the system. This will be allowed in the new steady-state initialization logic, however, the sum continuity equation will be evaluated for each volume to detect any initial flow imbalances that may exist prior to starting any steady-state iteration. Optionally, the user will be able to specify a minimum number of mass flow rates and have the code propagate those flow rates around the rest of the system. In this instance, only a single flow rate need be input in a loop and the sum continuity equation will be used to propagate it around the rest of the loop. In addition to the loop flow specification, the flow rates at all flow splits will have to be input. In all cases, if a flow imbalance is found, an error message will be written and the run terminated.

In some instances, the user may know the $\Delta \mathrm{P}$ across a channel better than the flow rate. Optionally, the steady-state momentum equation solution method could be extended to allow the user to supply a $\Delta P$ across a single component or several parallel components and have the sum momentum equation compute the flow rates and the pressure distribution within the components.

\subsubsection{Vapor Void Fraction}

In nonequilibrium volumes, the vapor void fraction is required input to define the initial thermodynamic state. With steady-state initialization, the user will be limited to fixing the fluid internal energy in a single-phase volume. The vapor void fraction values supplied for nonequilibrium volumes will be used for a first guess only and will not remain fixed during the steady-state iteration process.

\subsubsection{Initial Volume State Properties With Noncondensibles}

During normal operating conditions in LWRs, noncondensibles should not be flowing through the system. Noncondensibles will exist in isolated areas such as the accumulator or other volumes that will not be an integral part of the initialization process. Therefore, it will not be necessary to consider during the steady-state initialization process.

\subsubsection{Required User Input}

As stated earlier, current RELAP5 users must input the thermodynamic conditions for all volumes and much other information that will could be computed by steady-state initialization. These input conditions will also be required for the new steady-state initialization for three reasons. First, it will be desirable to use the steady-state initialization option with existing input models without major revisions to those input models. Secondly, the current input will provide guesses for the initial conditions to start the steady-state iterations. (Note that these initial guesses need not be very accurate and for new input models the user could easily provide these "initial estimates".) Thirdly, if the existing input requirements were revised, it would require a significant effort to revise the input coding. Given the current input structure will remain the same, only a small amount of 
additional input will be required for the user to specify which input parameters are to remain fixed when the steady-state initialization option is used.

The pressure distribution around the system is determined by the steady-state solution of the sum momentum equation. The user has the choice of fixing a mix of volume pressures or junction loss coefficients. There is a unique number of these parameters that must be fixed since one unknown parameter must be left to balance each momentum equation. If not enough parameters are fixed, the system is under specified and if too many are fixed, the system is over specified. As an example of the type of input required, consider a RELAP5 model of a straight pipe made up of several consecutive volumes and junctions. As one extreme, the user can fix the pressure in all the fluid volumes and have the code compute all the junction loss coefficients. For the other extreme, only one volume pressure is fixed, all but one junction loss coefficient are fixed and the remaining volume pressures would be computed. The user will be able to specify a mix of volume pressures and loss coefficients as long as there is a fixed loss coefficient for each unknown pressure or vice versa. This simple example illustrates how the user can choose to fix the parameters known best and allow the steady state to compute the rest.

The system energy distribution is determined by solution of the phasic energy equations. For steady-state initialization, the user must fix the internal energy in one single-phase fluid volume within a flow network. The internal energy of the rest of the fluid volumes will be computed by solving the coupled set of energy equations. As an example, consider a RELAP5 model of a PWR. To initialize the primary side fluid volumes, the internal energy need be fixed in a single volume such as a hot leg volume, a cold leg volume or any primary volume as long it was initially single phase. Since the primary and secondary side are not connected by flow paths, the secondary cannot be initialized unless the internal energy in a single volume is fixed there also. In addition, any stagnant branches (no flow initially) off of the flow networks in which the internal energy may be different than the volume they are connected to (such as the surge line and pressurizer), the internal energy will have to be fixed. Therefore, the typical PWR system model would require a fixed volume internal energy on the primary, the secondary and any stagnant legs.

As a minimum the sum continuity equation will be used to propagate unspecified junction flow rates and check the continuity balance in each fluid volume. The input requirement will be minimal in that only one junction flow rate is required for each simple pipe geometry and flow rates at splits must be defined (such as tees, manifolds or other complex geometries). Since the continuity equation is used only to propagate input flow values through the system, it can be solved separately from the others. Optionally, unknown flow rates can be computed from the momentum equations given a $\Delta \mathrm{P}$ across a channel or loop.

\subsection{Nearly Implicit Solution Method}

Experience obtained while developing steady-state solution schemes for other transient thermalhydraulic analysis codes (Refs. 2, 3, and 4), dictates that implicit techniques have much better convergence characteristics than explicit techniques. A fully implicit scheme with approximately $7 n$ globally coupled equations would converge well (n is the number of junctions or nodes). However, the current effort will use a less implicit method. It is based on the nearly implicit solution that is 
used in RELAP5 and has been reported to work well for the transient solution (Ref. 1). The nearly implicit method has the advantage of significantly reducing the CPU time required to obtain a solution in addition to the fact that the steady-state development effort can be reduced by using much of the existing coding for the nearly implicit method.

The steady-state solution will employ an iterative scheme, where the iterative loop will be similar to the time advancement loop in the transient solution. The starting point lor zeroth iterate system state) for the iterative solution will correspond to the initial condition specifications that are currently supplied with the input model.

The steady state will use a two-step iteration scheme that closely follows the transient scheme, making use of the various steps that increase numerical efficiency and reduce computational effort. The nearly implicit solution method solves the sum and difference momentum equations as a globally coupled system of $2 n$ equations ( $n=$ number of junctions). However, the global system of equations initially contains $2 n+m$ unknowns ( $m=$ number of control volumes). A locally coupled set of five continuity and energy equations is written for each control volume. The intermediate solutions for these local equation sets gives a relationship for the control volume pressure in terms of the associated phasic velocities. These relationships are substituted into the $2 \mathrm{n}$ coupled equations to eliminate the pressure dependency. Once this is accomplished, the velocities are obtained by using a direct solution method.

The next step evaluates the pressures and the intermediate or "tilde" level values for the phasic energies and densities. They are then used to evaluate "tilde" level mass and energy transfer rate source terms that are used in the final evaluation of the mass and energy equations.

In the final step, the mass and energy equations for each phase are cast in separate, but similar, systems of equations. The coefficient matrices for the mass and energy equations are identical but the solution vector and RHS are different. Thus, only one matrix of order $m$ must be inverted for each phase. After the inverse is obtained, it can be used to obtain the solution for both the mass and energy equation solutions. This significantly reduces the CPU time since only two order $\mathrm{m}$ matrices must be inverted rather than one order $5 \mathrm{~m}$ matrix.

The application of the nearly implicit method for the steady-state solution testbed code has some variations from the transient solution. For the single-phase situation considered in the testbed code, the "tilde" level solution has no effect on the first step solution and is thus neglected. It may be necessary to incorporate the intermediate step to obtain "tilde" level values of vapor void fraction for two-phase situations.

The sum and difference momentum equations used in the first step are given in steady-state form. The sum momentum equation is obtained by adding Equations 3-7 and 3-8 and dividing by the area that appears in each term. The sum momentum equation 


$$
\begin{aligned}
& \frac{1}{2} \alpha_{0} \rho_{g} \frac{\partial v_{g}^{2}}{\partial x}+\frac{1}{2} \alpha_{f} \rho_{f} \frac{\partial v_{f}^{2}}{\partial x}=-\alpha_{d} \frac{\partial P}{\partial x}-\alpha_{f} \frac{\partial P}{\partial x}+\alpha_{0} \rho_{0} B_{x}+\alpha_{f} \rho_{f} B_{x}-\alpha_{0} \rho_{0} f w g v_{g} \\
& -\alpha_{f} \rho_{f} f_{w f} v_{f}-\Gamma_{0}\left(v_{0}-v_{f}\right)-\alpha_{0} \rho_{0} H_{t g} v_{0}-\alpha_{f} \rho_{f} H_{f} v_{f}-\Gamma_{n c} v_{0}-\Gamma_{s} v_{f}
\end{aligned}
$$

Before subtracting Equation 3-8 from Equation 3-7 to obtain the difference form, first divide the vapor momentum Equation 3-7 by $\alpha_{a} \rho_{g}$, and the fluid momentum Equation 3-8 by $\alpha_{f} \rho_{f}$. Then subtract Equation 3-8 from Equation 3-7, dividing by the area, to obtain the difference momentum equation

$$
\begin{aligned}
& \frac{1}{2}\left(\frac{\alpha_{0} \rho_{o}}{\alpha_{0} \rho_{0}}\right) \frac{\partial v_{o}^{2}}{\partial x^{2}}-\frac{1}{2}\left(\frac{\alpha_{f} \rho_{f}}{\alpha_{f} \rho_{f}}\right) \frac{\partial v_{f}^{2}}{\partial x}=-\left(\frac{1}{\rho_{g}}-\frac{1}{\rho_{f}}\right) \frac{\partial P}{\partial x}-\left[\left(1-f_{x}\right)+\alpha_{g} f_{x}\left(1-\rho_{g} / \rho_{f}\right)\right] f_{w o} v_{g} \\
& +\left[\left(1-f_{x}\right)+\alpha_{f} f_{x}\left(1-\rho_{f} / \rho_{g}\right)\right] f_{w f} v_{f}-\rho_{1} F_{1}\left(C_{1} v_{0}-C_{0} v_{f}\right)+\frac{\rho_{1} \Gamma_{g}\left(v_{g}-v_{f}\right)}{\alpha_{0} \rho_{g} \alpha_{f} \rho_{f}} \\
& -\left(\frac{\alpha_{g} \rho_{g}}{\alpha_{g} \rho_{g}}\right) H_{s} v_{g}+\left(\frac{\alpha_{f} \rho_{f}}{\alpha_{f} \rho_{f}}\right) H_{f f} v_{f}-\frac{\Gamma_{n c} v_{g}}{\alpha_{g} \rho_{g}}+\frac{\Gamma_{s} v_{f}}{\alpha_{f} \rho_{f}}
\end{aligned}
$$

where the relationships

$$
\alpha_{g} \rho_{g} f_{1 g}=\alpha_{f} \rho_{f} f_{1 f}=\alpha_{g} \rho_{g} \alpha_{f} \rho_{f} F_{1}
$$

where

$\Gamma_{0}=-\frac{H_{10}\left(T_{s}-T_{0}\right)+H_{1 f}\left(T_{s}-T_{f}\right)}{h_{g}^{*}-h_{f}^{*}}+\Gamma_{w}$

have been used.

The spatial noding and integration of the field equations is discussed in Reference 1. It is noted that the equations are developed for slowly changing areas (stream tube approach). However, special models are included to compensate for special effects (abrupt area changes, choking, special flow patterns, etc.). The mass and energy equations are integrated from $x_{1}$ to $x_{1+1}$, defining volume boundaries. The momentum equations are integrated from volume (cell) center to volume center.

For the nearly implicit formulation, an extra expansion is made for the momentum flux term. First, the differencing of the momentum term is

$$
\frac{1}{2} \frac{\partial v_{0}^{2}}{\partial x}-\frac{1}{2} \frac{\partial v_{f}^{2}}{\partial x}-\frac{1}{2}\left[\left(v_{0 . L}\right)^{2}-\left(v_{0 . K}\right)^{2}\right]
$$


which shows that the velocities are written in terms of volume flows, whereas the computation is written in terms of velocities at the cell or volume edges. Also, for time advancement schemes or steady-state iteration, the terms must be linearized. RELAP5 uses the following formulation to approximate the momentum flux terms, which is also used for steady state.

$\left(v_{0, L}^{m+1}\right)^{2}-\left[v_{0, L}^{m+1}-v_{0, L}^{m}\right]^{2}+2 v_{0, L}^{m}\left[v_{0, L}^{m+1}-v_{0, L}^{m}\right]+\left[v_{0, L}^{m}\right]^{2}$

The leading quadratic term is dropped, so that the expression is linear in the new iteration $(m+1)$ or time advancement $(\mathrm{n})$, as

$\left(v_{0, L}^{m+1}\right)^{2}-2 v_{0, L}^{m}\left[v_{0, L}^{m+1}-v_{0, L}^{m}\right]+\left[v_{0, L}^{m}\right]^{2}$

Rather than writing the equations twice, first showing the limits of integration for each term, and then writing the final form, the final form of the differenced equations is shown directly. For the sum momentum equation, the finite difference form of Equation 3-11 is

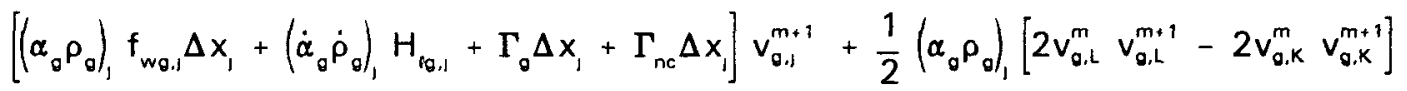

$$
\begin{aligned}
& +\left[\left(\alpha_{f} \rho_{f}\right)_{1} f_{w f, j} \Delta x_{t}+\left(\dot{\alpha}_{f} \dot{\rho}_{f}\right)_{1} H_{t f, j}-\Gamma_{\delta} \Delta x_{1}+\Gamma_{s} \Delta x_{f}\right] v_{f, 1}^{m+1} \\
& +\frac{1}{2}\left(\alpha_{t} \rho_{f}\right)_{1}\left[2 v_{f, L}^{m} v_{f, L}^{m+1}-2 v_{f, K}^{m} v_{f, K}^{m+1}\right]=\left(P_{k}^{m+1}-P_{L}^{m+1}\right)+\frac{1}{2}\left(\alpha_{0} \rho_{0}\right)\left[\left(v_{0, L}^{m}\right)^{2}-\left(v_{g, k}^{m}\right)^{2}\right] \\
& +\frac{1}{2}\left(\alpha_{f} \rho_{f}\right)_{1}\left[\left(v_{f, L}^{m}\right)^{2}-\left(v_{t, k}^{m}\right)^{2}\right]+\rho_{1} B_{x} \Delta x_{1}
\end{aligned}
$$

The finite difference form for the difference momentum equation, Equation 3-12 is

$$
\begin{aligned}
& {\left[\left[\left(1-f_{x}\right)+\alpha_{f, j} f_{x}\left(1-\rho_{f} / \rho_{0}\right)_{1}\right] f_{w f, j} \Delta x_{1}+\left(\frac{\dot{\alpha}_{f} \dot{\rho}_{f}}{\alpha_{f} \rho_{f}}\right)_{1} H_{f f, j}+\rho_{f} F_{i, j} C_{0} \Delta x_{1}+\frac{\rho_{1} \Gamma_{0} \Delta x_{J}}{\left(\alpha_{0} \rho_{g} \alpha_{f} \rho_{f}\right)_{1}}+\frac{\Gamma_{s} \Delta x_{j}}{\left(\alpha_{f} \rho_{f}\right)_{1}}\right] v_{f, j}^{m+1}} \\
& +\frac{1}{2}\left(\frac{\dot{\alpha}_{f} \dot{\rho}_{f}}{\alpha_{f} \rho_{f}}\right)\left[2 v_{f, L}^{m} v_{f, L}^{m+1}-2 v_{f, k}^{m} v_{f, K}^{m+1}\right]-\left[\left[\left(1-f_{x}\right)+\alpha_{g, t} f_{x}\left(1-\rho_{o} / \rho_{f}\right)_{1}\right] f_{w g, l} \Delta x_{f}+\left(\frac{\dot{\alpha}_{0} \dot{\rho}_{0}}{\alpha_{0} \rho_{0}}\right)_{1} H_{t g, 1}\right. \\
& \left.+\rho_{1} F_{1,1} C_{1} \Delta x_{1}+\frac{\rho_{1} \Gamma_{\theta} \Delta x_{1}}{\left(\alpha_{0} \rho_{0} \alpha_{f} \rho_{f}\right)_{1}}+\frac{\Gamma_{n c} \Delta x_{1}}{\left(\alpha_{0} \rho_{0}\right)_{1}}\right] v_{0,1}^{m+1}-\frac{1}{2}\left(\frac{\dot{\alpha}_{0} \dot{\rho}_{0}}{\alpha_{0} \rho_{0}}\right)_{1}\left[2 v_{0, L}^{m} v_{0, L}^{m+1}-2 v_{0, K}^{m} v_{g, K}^{m+1}\right] \\
& =-\left(\frac{\rho_{g}-\rho_{f}}{\rho_{g} \rho_{f}}\right)_{1}\left(P_{L}^{m+1}-P_{K}^{m+1}\right)+\frac{1}{2}\left(\frac{\dot{\alpha}_{f} \dot{\rho}_{f}}{\alpha_{f} \rho_{f}}\right)\left[\left(v_{f, L}^{m}\right)^{2}-\left(v_{f, K}^{m}\right)^{2}\right]-\frac{1}{2}\left(\frac{\dot{\alpha}_{g} \dot{\rho}_{g}}{\alpha_{0} \rho_{g}}\right)\left[\left(v_{g, L}^{m}\right)^{2}-\left(v_{g, K}^{m}\right)^{2}\right]
\end{aligned}
$$


where

$f_{x}=$ a weighting constant, $0 \leq f_{x} \leq 1$

which is determined by the form of the friction model.

Comparing Equation 3-19 to Equation 3-12, note that Equation 3-19 has been multiplied through by $(-1)$ to put it in the same form as found in the RELAP5 coding. The - superscript indicates donor junction values as opposed to junction average values.

Also, in the momentum flux terms, the product terms $v^{m} v^{m+1}$ are global and can be written more rigorously as

$\sum_{j} v_{k}^{m} v_{k}^{m+1}$

where the summation is over all junctions connected to the corresponding volume.

For the transient solution method, the pressures are eliminated from the sum and difference momentum equations by using the intermediate local pressure solution. Here, we use the sum momentum equation to obtain the volume pressures and the difference momentum equation to obtain the vapor velocity. At this point, there are more unknowns than equations since both equations are written in terms of the liquid velocity $v_{f}$. The following relationship, obtained from the definition of the mass flow rate, can be used to eliminate the liquid velocity. The junction liquid velocity is given by

$v_{t, 1}=\frac{w_{1}-\left(\alpha_{g} \rho_{0} v_{g} A\right)_{J}}{\left(\alpha_{f} \rho_{f} A\right)_{1}}$

which uses the mass flow rate initial condition $w_{1}$.

Given that flow initial conditions are used in the testbed code, there are generally fewer pressures to be determined than sum momentum equations. There must be additional unknown(s) to satisfy the sum momentum balance. Users typically adjust form loss coefficients to obtain desired pressure drops, so a loss coefficient is included as an unknown. For the analysis performed with the testbed code, the sum momentum equations are used to compute the downstream pressure and the exit loss coefficient is computed using the outlet junction momentum equation. A production code will require that any given sum momentum equation can be used to compute either the upstream or downstream pressure, or the loss coefficient associated with the junctions. Additional logic will be required to determine the correct unknown for the given user input as is done in the RETRAN codes [Refs. 2 and 3]. 
After the set of sum and difference equations are solved for the new iterate values of $\mathrm{P}^{m+1}$ and/or $F_{k}^{m+1}$ and $v_{0}^{m+1}$, the fluid velocity is obtained from the above relationship.

The first step is to eliminate $v_{f}^{m+1}$ from Equations 3-18 and 3-19 in terms of $w, v_{o}^{m+1}$. Substitution in the sum momentum equation yields

$$
\begin{aligned}
& {\left[\left(\alpha_{0} \rho_{s}\right)_{1} f_{w 0.1} \Delta x_{1}+\left(\dot{\alpha}_{0} \dot{\rho}_{0}\right)_{1} H_{10.1}+\Gamma_{0} \Delta x_{1}+\Gamma_{n c} \Delta x_{1}\right] v_{0.1}^{m+1}} \\
& +\left[\left(\alpha_{1} \rho_{t}\right)_{1} f_{w f, j} \Delta x_{1}+\left(\dot{\alpha}_{f} \dot{\rho}_{f}\right)_{1} H_{f, t}-\Gamma_{0} \Delta x_{1}+\Gamma_{s} \Delta x_{1}\right]\left[\frac{w_{1}^{m}-\left(\alpha_{0} \rho_{0} A\right)_{1} v_{0,1}^{m+1}}{\left(\alpha_{f} \rho_{f} A\right)_{1}}\right] \\
& +\frac{1}{2}\left(\alpha_{\theta} \rho_{0}\right)_{1}\left[2 v_{0, L}^{m} v_{0, L}^{m+1}-2 v_{0, K}^{m} v_{0, K}^{m+1}\right] \\
& +\frac{1}{2}\left(\alpha_{f} \rho_{f}\right)_{1}\left[2 v_{f, L}^{m}\left\{\frac{w_{1}^{m}-\left(\alpha_{0} \rho_{g} A\right)_{1} v_{0, L}^{m+1}}{\left(\alpha_{f} \rho_{f} A\right)_{1}}\right\}-2 v_{f, K}^{m}\left\{\frac{w_{1}^{m}-\left(\alpha_{0} \rho_{0} A\right)_{1} v_{0, K}^{m+1}}{\left(\alpha_{f} \rho_{t} A\right)_{1}}\right\}\right] \\
& =\left(P_{k}^{m+1}-P_{L}^{m+1}\right)+\frac{1}{2}\left(\alpha_{0} \rho_{0}\right)_{1}\left[\left(v_{0, L}^{m}\right)^{2}-\left(v_{0, k}^{m}\right)^{2}\right]+\frac{1}{2}\left(\alpha_{t} \rho_{f}\right)_{1}\left[\left(v_{f, L}^{m}\right)^{2}-\left(v_{f, k}^{m}\right)^{2}\right]+\rho_{1} B_{x} \Delta x_{1}
\end{aligned}
$$

This equation is now arranged, putting all $m+1$ pieces on the LHS and putting all old iterate values $(\mathrm{m})$ on the RHS. 


$$
\begin{aligned}
& \left(P_{L}^{m+1}-P_{k}^{m+1}\right)+\left\{\left[\left(\alpha_{0} \rho_{0}\right)_{1} f_{w_{0,1}} \Delta x_{1}+\left(\dot{\alpha}_{0} \dot{\rho}_{0}\right)_{1} H_{b, 1}+\left(\Gamma_{0}+\Gamma_{n c}\right) \Delta x_{b}\right]\right. \\
& \left.-\left(\frac{\alpha_{0} \rho_{0}}{\alpha_{f} \rho_{f}}\right)_{1}\left[\left(\alpha_{f} \rho_{f}\right)_{1} f_{w f, 1} \Delta x_{1}+\left(\dot{\alpha}_{f} \dot{\rho}_{f}\right)_{1} H_{f f, 1}-\left(\Gamma_{0}-\Gamma_{s}\right) \Delta x_{1}\right]\right\} v_{0,1}^{m+1} \\
& +\frac{1}{2}\left\{\left(\alpha_{0} \rho_{0}\right)_{1} 2 v_{g, L}^{m} v_{0, L}^{m+1}-\left(\alpha_{f} \rho_{f}\right) 2 v_{f, L}^{m}\left(\frac{\alpha_{0} \rho_{0}}{\alpha_{f} \rho_{f}}\right)_{1} v_{0, L}^{m+1}\right\} \\
& -\frac{1}{2}\left\{\left(\alpha_{g} \rho_{o}\right)_{1} 2 v_{g, k}^{m} v_{g, K}^{m+1}-\left(\alpha_{f} \rho_{f}\right) 2 v_{f, K}^{m}\left(\frac{\alpha_{g} \rho_{g}}{\alpha_{f} \rho_{f}}\right)_{1} v_{g, K}^{m+1}\right\} \\
& -\frac{1}{2}\left(\alpha_{0} \rho_{g}\right)_{j}\left[\left(v_{0, L}^{m}\right)^{2}-\left(v_{g, K}^{m}\right)^{2}\right]+\frac{1}{2}\left(\alpha_{f} \rho_{f}\right)_{j}\left[\left(v_{f, L}^{m}\right)^{2}-\left(v_{f, K}^{m}\right)^{2}\right] \\
& +\rho_{1} B_{x} \Delta x_{1}-\frac{1}{2}\left(\alpha_{t} \rho_{t}\right)_{1} 2 v_{f, L}^{m}\left[\frac{w_{1}}{\left(\alpha, \rho_{t} A\right)}\right]_{L}+\frac{1}{2}\left(\alpha_{t} \rho_{f}\right)_{1} 2 v_{f, K}^{m}\left[\frac{w_{1}}{\left.\left(\alpha, \rho_{f} A\right)_{1}\right]_{k}}\right. \\
& -\left[\left(\alpha_{f} \rho_{f}\right)_{1} f_{w t, 1} \Delta x_{1}+\left(\dot{\alpha}_{t} \dot{\rho}_{t}\right)_{1} H_{t f, j}-\left(\Gamma_{0}-\Gamma_{s}\right) \Delta x_{1}\right]\left[\frac{w_{1}}{\left(\alpha_{f} \rho_{f} A\right)}\right]
\end{aligned}
$$

For the above equation, the subscripts $L, K$ for $v_{0}^{m+1}$ in momentum flux term indicate the velocities are sums of junction velocities. This also occurs on the RHS for momentum flux pieces that have $w_{1}$ multipliers, i.e., the junction mass flow terms are summed over the junctions.

Making the same substitutions in the difference momentum equation (Eq. 3-19) and moving pieces to the LHS or RHS, gives 


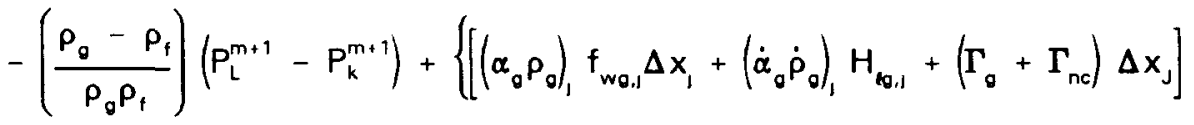

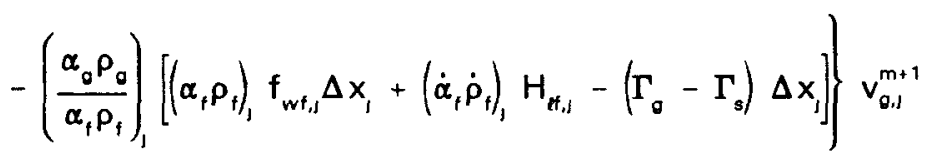

$$
\begin{aligned}
& +\frac{1}{2}\left\{\left(\alpha_{\theta} \rho_{g}\right)_{,} 2 v_{g, L}^{m} v_{g, L}^{m+1}-\left(\alpha_{f} \rho_{f}\right)_{,} 2 v_{f, L}^{m}\left(\frac{\alpha_{g} \rho_{g}}{\alpha_{f} \rho_{f}}\right)_{1} v_{g, L}^{m+1}\right\} \\
& -\frac{1}{2}\left\{\left(\alpha_{\theta} \rho_{g}\right)_{1} 2 v_{0, K}^{m} v_{a, K}^{m+1}-\left(\alpha_{f} \rho_{f}\right)_{1} 2 v_{f, K}^{m}\left(\frac{\alpha_{\theta} \rho_{g}}{\alpha_{f} \rho_{f}}\right)_{j} v_{0, K}^{m+1}\right\} \\
& =\frac{1}{2}\left(\alpha_{g} \rho_{g}\right)_{1}\left[\left(v_{g, L}^{m}\right)^{2}-\left(v_{g, K}^{m}\right)^{2}\right]+\frac{1}{2}\left(\alpha_{f} \rho_{f}\right)_{j}\left[\left(v_{f, L}^{m}\right)^{2}-\left(v_{f, K}^{m}\right)^{2}\right] \\
& +\rho_{1} B_{x} \Delta x_{1}-\frac{1}{2}\left(\alpha_{f} \rho_{f}\right)_{1} 2 v_{f, L}^{m}\left[\frac{w_{f}}{\left(\alpha_{f} \rho_{f} A\right)}\right]_{1}+\frac{1}{2}\left(\alpha_{f} \rho_{f}\right)_{1} 2 v_{f, K}^{m}\left[\frac{w_{1}}{\left(\alpha_{f} \rho_{f} A\right)_{1}}\right]_{K} \\
& -\left[\left(\alpha_{f} \rho_{f}\right)_{1} f_{w f, s} \Delta x_{1}+\left(\dot{\alpha}_{f} \dot{\rho}_{f}\right)_{1} H_{t f, 1}-\left(\Gamma_{o}-\Gamma_{s}\right) \Delta x_{1}\right]\left[\frac{w_{1}}{\left(\alpha_{f} \rho_{f} A\right)_{1}}\right]
\end{aligned}
$$

In Equations 3-23 and 3-24, the new time variables are $P_{K, L}^{m+1}, v_{0,1}^{m+1}$, or the pressures and gas velocities around the loop.

The user can optionally allow the junction loss coefficient to be computed rather than a volume pressure. The sum and difference momentum equations are still used, but for this case, the dependent variables will be $F_{K, 1}^{m+1}$ and $v_{g, 1}^{m+1}$. To accomplish this, first examine the coding for the computation of the loss coefficient (user input $F_{f}, F_{r}$ ). This term is

$$
\begin{aligned}
& H_{t f, j}=\left(\left|v_{f}\right|+0.01 A_{\text {throat }}\right)_{j}\left(\frac{1}{2} \frac{\dot{\alpha}_{f} \dot{\rho}_{f}}{\alpha_{f} \rho_{f}}\right)\left[F_{\text {ormft }}+F_{\text {losst }}+F_{\text {let }}\right] \\
& H_{\text {tg, } 1}=\left(\left|v_{g}\right|+0.01 A_{\text {throat }}\right)_{1}\left(\frac{1}{2} \frac{\dot{\alpha}_{g} \dot{\rho}_{g}}{\alpha_{g} \rho_{g}}\right)\left[F_{\text {ormgl }}+F_{\text {lossg }}+F_{\text {let }}\right]
\end{aligned}
$$

and

$$
F_{\text {lossf }}=F_{\text {lossg }}= \begin{cases}F_{\text {lunf }} \text { for } v_{g^{\prime}} & v_{f}>0 \\ F_{\text {lunr }} \text { for } v_{g^{\prime}} & v_{f}<0\end{cases}
$$


Define coefficients

$$
\begin{aligned}
& C_{f s}=\left(\left|v_{f}\right|+0.01 A_{\text {throat }}\right)_{1}\left(\frac{1}{2} \frac{\dot{\alpha}_{f} \dot{\rho}_{f}}{\alpha_{f} \rho_{f}}\right) \\
& C_{o s}=\left(\left|v_{g}\right|+0.01 A_{\text {throat }}\right)_{1}\left(\frac{1}{2} \frac{\dot{\alpha}_{g} \dot{\rho}_{g}}{\alpha_{g} \rho_{g}}\right)
\end{aligned}
$$

and then write

$$
\begin{aligned}
& H_{f f, j}-C_{f s}\left(F_{\text {ormf(y) }}+F_{\text {lossf(y) }}+F_{\text {(et( }(j)}\right) \\
& H_{\text {po, }}-C_{0 s}\left(F_{\text {ormg(1) }}+F_{\text {losso(1) }}+F_{\text {jet(1) }}\right)
\end{aligned}
$$

Similar to solving for pressure at the new iterate, now solve for the loss coefficient at the new iterate. This will introduce the product term

$$
F_{\text {lunf }}^{m+1} v_{0.1}^{m+1}=F_{\text {loss }(1)} v_{0.1}
$$

This term is expanded as

$$
F_{\text {junt }}^{m}\left(v_{0.1}^{m+1}-v_{0.1}^{m}\right)+\left(F_{\text {junf }}^{m+1}-F_{\text {junt }}^{m}\right) v_{0.1}^{m}
$$

which yields

$$
F_{\text {lunf }}^{m} v_{0,1}^{m+1}+v_{0,1}^{m} F_{\text {iunf }}^{m+1}-2 F_{\text {lunf }}^{m} v_{0,1}^{m}
$$

This expansion will give additional terms to the LHS of the equations and also additional terms to the RHS. A loss coefficient also appears on the RHS, and when put at the new iterate level, it will appear on the LHS. Also, as a coding convenience, the old time piece on the RHS will need to be factored out.

Making the substitutions from Equations 3-27a, 3-27b, and 3-28b into the sum and difference equations, and collecting terms, yields the equation set for junctions where it is specified that a loss coefficient is to be computed. Note that in the demonstration analyses, the outlet junction downstream pressure is known when solving for $F_{K}$. 
For the sum momentum equation

$$
\begin{aligned}
& -P_{k}^{m+1}+\left\{\left(\alpha_{0} \rho_{0}\right)_{1} f_{w \sigma, 1} \Delta x_{1}+\left(\dot{\alpha}_{0} \dot{\rho}_{0}\right)_{1} H_{t o, 1}+\left(\Gamma_{0}+\Gamma_{n c}\right) \Delta x_{1}\right] \\
& \left.-\left(\frac{\alpha_{0} \rho_{0}}{\alpha_{t} \rho_{f}}\right)_{1}\left[\left(\alpha_{f} \rho_{f}\right)_{1} f_{w t, t} \Delta x_{1}+\left(\dot{\alpha}_{t} \dot{\rho}_{t}\right)_{1} H_{t, 1}-\left(\Gamma_{0}-\Gamma_{s}\right) \Delta x_{1}\right]\right\} v_{0,1}^{m+1}
\end{aligned}
$$

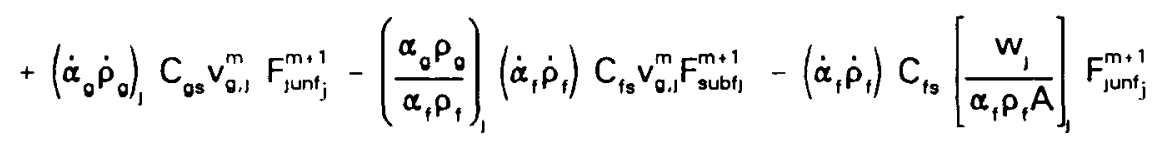

$$
\begin{aligned}
& +\frac{1}{2}\left\{\left(\alpha_{\diamond} \rho_{0}\right)_{j} 2 v_{g, L}^{m} v_{g, L}^{m+1}-\left(\alpha_{t} \rho_{f}\right)_{,} 2 v_{t, L}^{m}\left(\frac{\alpha_{g} \rho_{g}}{\alpha_{t} \rho_{f}}\right)_{1} v_{g, L}^{m+1}\right\} \\
& -\frac{1}{2}\left\{\left(\alpha_{s} \rho_{0}\right)_{,} 2 v_{g, K}^{m} v_{0, K}^{m+1}-\left(\alpha_{t} \rho_{f}\right)_{,} 2 v_{f, K}^{m}\left(\frac{\alpha_{o} \rho_{g}}{\alpha_{t} \rho_{f}}\right)_{1} v_{g, k}^{m+1}\right\} \\
& -\frac{1}{2}\left(\alpha_{\theta} \rho_{\sigma}\right)_{1}\left[\left(v_{0, L}^{m}\right)^{2}-\left(v_{\theta, K}^{m}\right)^{2}\right]+\frac{1}{2}\left(\alpha_{f} \rho_{f}\right)_{1}\left[\left(v_{f, L}^{m}\right)^{2}-\left(v_{t, K}^{m}\right)^{2}\right] \\
& -\left[\left(\alpha_{t} \rho_{t}\right)_{1} f_{w t, 1} \Delta x_{t}+\left(\dot{\alpha}_{t} \dot{\rho}_{t}\right)_{1} H_{t, t,}-\left(\Gamma_{\theta}-\Gamma_{s}\right) \Delta x_{1}\right]\left[\frac{w}{\alpha_{t} \rho_{t} A}\right]_{1} \\
& -\frac{1}{2}\left(\alpha_{t}, \rho_{t}\right)_{1} 2 v_{t, L}^{m}\left[\frac{w_{1}}{\left(\alpha_{t} \rho_{t} A\right)_{t}}\right]_{L}+\frac{1}{2}\left(\alpha_{t}, \rho_{t}\right)_{1} 2 v_{t, K}^{m}\left[\frac{w_{1}}{\left(\alpha_{t} \rho_{t} A\right)_{1}}\right]_{K}
\end{aligned}
$$

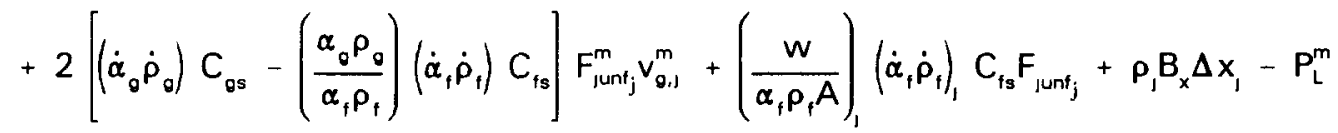


For the difference momentum equation

$$
\begin{aligned}
& -\left(\frac{\rho_{0}-\rho_{f}}{\rho_{0} \rho_{f}}\right)_{K} P_{K}^{m+1}+\left\{\left[\left(\alpha_{0} \rho_{0}\right)_{1} f_{w 0,1} \Delta x_{1}+\left(\dot{\alpha}_{0} \dot{\rho}_{0}\right)_{1} H_{(0,1)}+\left(\Gamma_{0}+\Gamma_{n c}\right) \Delta x_{1}\right]\right. \\
& \left.-\left(\frac{\alpha_{\theta} \rho_{g}}{\alpha_{f} \rho_{f}}\right)_{1}\left[\left(\alpha_{f} \rho_{f}\right)_{,} f_{w f, 1} \Delta x_{f}+\left(\dot{\alpha}_{f} \dot{\rho}_{f}\right)_{1} H_{t f, 1}-\left(\Gamma_{\theta}-\Gamma_{s}\right) \Delta x_{1}\right]\right\} v_{0,1}^{m+1} \\
& -\left[C_{g s} v_{0, j}^{m}+\left(\frac{\alpha_{g} \rho_{s}}{\alpha_{f} \rho_{f}}\right) C_{f s} v_{g, t}^{m}-\left(\frac{w}{\alpha_{f} \rho_{f} A}\right)_{j} C_{f s}\right] f_{f u n f}^{m+1} \\
& +\frac{1}{2}\left\{\left(\alpha_{o} \rho_{f}\right)_{,} 2 v_{0, L}^{m} v_{g, L}^{m+1}-\left(\alpha_{f} \rho_{f}\right)_{,} 2 v_{f, L}^{m}\left(\frac{\alpha_{0} \rho_{0}}{\alpha_{f} \rho_{f}}\right)_{1} v_{g, L}^{m+1}\right\} \\
& -\frac{1}{2}\left\{\left(\alpha_{0} \rho_{0}\right)_{1} 2 v_{0, K}^{m} v_{0, K}^{m+1}-\left(\alpha_{t} \rho_{t}\right)_{1} 2 v_{t, K}^{m}\left(\frac{\alpha_{0} \rho_{0}}{\alpha_{f} \rho_{f}}\right) v_{0, K}^{m+1}\right\} \\
& =\frac{1}{2}\left(\alpha_{\theta} \rho_{s}\right)_{1}\left[\left(v_{s, L}^{m}\right)^{2}-\left(v_{\theta, K}^{m}\right)^{2}\right]+\frac{1}{2}\left(\alpha_{t} \rho_{f}\right)_{1}\left[\left(v_{f, L}^{m}\right)^{2}-\left(v_{f, K}^{m}\right)^{2}\right] \\
& +\rho_{t} B_{x} \Delta x_{1}-\frac{1}{2}\left(\alpha_{f} \rho_{f}\right)_{1} 2 v_{f, L}^{m}\left[\frac{w_{1}}{\left(\alpha_{f} \rho_{f} A\right)_{,}}\right]_{L}+\frac{1}{2}\left(\alpha_{f} \rho_{f}\right)_{1} 2 v_{f, K}^{m}\left[\frac{w_{1}}{\left\langle\alpha_{f} \rho_{f} A\right\rangle_{1}}\right]_{k} \\
& -\left[\left(\alpha_{f} \rho_{f}\right)_{1} f_{w f, 1} \Delta x_{1}+\left(\dot{\alpha}_{f} \dot{\rho}_{f}\right)_{1} H_{n_{f, 1}}-\left(\Gamma_{0}-\Gamma_{s}\right) \Delta x_{1}\right]\left[\frac{w}{\alpha_{f} \rho_{f} A}\right]_{J} \\
& -2\left[C_{g s}+\left(\frac{\alpha_{g} \rho_{g}}{\alpha_{f} \rho_{f}}\right) C_{f s}\right] v_{g, 1}^{m} F_{j u n f_{j}}^{m}+\left(\frac{w}{\alpha_{f} \rho_{f} A}\right)_{1} C_{f s} F_{j u n f}^{m}+\left(\frac{\rho_{g}-\rho_{f}}{\rho_{g} \rho_{f}}\right)_{L} P_{L}^{m}
\end{aligned}
$$

The sum and difference equations are solved by matrix inversion for a $2 n$ set of globally coupled equations using the same direct solver used for the transient nearly implicit method. For steady state, like the transient, this becomes the first step of the solution, which yields $P^{m+1}$ and/or $F_{\text {unf }}^{m+1}$, and $v_{g}^{m+1}$. The liquid velocities, $v_{f}$, are then determined using Equation 3-21.

The nearly implicit transient solution method updates the intermediate locally coupled mass and energy equations to obtain the "tilde" level values of the dependent variables. They are then used to update the mass and energy transfer rate terms to the "tilde" level by using first order Taylor series expansions. These "tilde" level source terms are then used in the second step solution of the phasic continuity and energy equations to stabilize the convective terms.

For the single-phase testbed code, the "tilde" level equations are degenerate and do not provide any coupling between the first and second steps. Therefore, the intermediate step has been neglected. 
It may, however, offer some useful coupling for the more general two-phase situation and should be included.

The transient second step solves the phasic density and energy equations, rather than the sum and difference density equations used in the intermediate step. This is true for the steady-state solution, with the exception of the sum density equation which is used in place of the liquid equation. By doing so, the flow initial conditions can be included in the solution while also ensuring that continuity is satisfied for both phases.

The finite difference form for the noncondensible density, the sum density and vapor density equations, and the phasic energy equations are given below.

The noncondensible density equation is

$\left(\dot{\alpha}_{0} \dot{\rho}_{\theta} X_{n} A\right)_{1+1}^{m+1} v_{0,1+1}^{m+1}-\left(\dot{\alpha}_{\theta} \dot{\rho}_{\theta} X_{n} A\right)_{1}^{m+1} v_{0.1}^{m+1}-\Gamma_{n c} V_{L}=0$

The vapor density equation in finite difference form is

$$
\begin{aligned}
\left(\dot{\alpha}_{0} \dot{\rho}_{0} A\right)_{1+1}^{m+1} v_{0, j+1}^{m+1} & -\left(\dot{\alpha}_{0} \dot{\rho}_{0} A\right)_{1}^{m+1} v_{0, t}^{m+1} \\
& +\left[\frac{V_{L}}{h_{0}^{*}-h_{f}^{*}}\right]\left[H_{1 d}\left(T_{s}-T_{0}\right)+H_{1 f}\left(T_{s}-T_{f}\right)\right]-V_{L} \Gamma_{w}-\Gamma_{n c} V_{L}=0
\end{aligned}
$$

while the vapor energy equation in finite difference form is

$$
\begin{aligned}
& \left(\dot{\alpha}_{0} \dot{\rho}_{0} U_{0}\right)_{1+1}^{m+1} A_{1+1} v_{0.1+1}^{m+1}-\left(\dot{\alpha}_{0} \dot{\rho}_{0} U_{0}\right)_{1}^{m+1} A_{, j} v_{0,1}^{m+1}+P\left(\dot{\alpha}_{0} A\right)_{j+1}^{m+1} v_{0,1+1}^{m+1}-P\left(\dot{\alpha}_{0} A\right)_{1}^{m+1} v_{0.1}^{m+1} \\
& +\left[\frac{h_{f}^{*} V_{L}}{h_{g}^{*}-h_{f}^{*}}\right] H_{10}\left(T_{s}-T_{d}\right)+\left[\frac{h_{g}^{*} V_{L}}{h_{g}^{*}-h_{f}^{*}}\right] H_{f f}\left(T_{s}-T_{f}\right) \\
& -\left[\left(\frac{1+\varepsilon}{2}\right) h_{g s}+\left(\frac{1-\varepsilon}{2}\right) h_{f s}\right] \Gamma_{w} V_{L}-O_{w g} V_{L}-\operatorname{Diss}_{g} V_{L}-\Gamma_{n c} H_{n c} V_{L}
\end{aligned}
$$

The finite difference form of the sum density equation is

$$
\left(\dot{\alpha}_{g} \dot{\rho}_{g} A\right)_{1+1}^{m+1} v_{g, t+1}^{m+1}+\left(\dot{\alpha}_{f} \dot{\rho}_{f} A\right)_{1+1}^{m+1} v_{f, t+1}^{m+1}-\left(\dot{\alpha}_{g} \dot{\rho}_{g} A\right)_{1}^{m+1} v_{g, 1}^{m+1}-\left(\dot{\alpha}_{f} \dot{\rho}_{f} A\right)_{1}^{m+1} v_{f, 1}^{m+1}-\Gamma_{n c} V_{L}-\Gamma_{s} V_{L}=0
$$


The finite difference form of the liquid energy equation is

$$
\begin{aligned}
& \left(\dot{\alpha}_{t} \dot{\rho}_{f} U_{f}\right)_{1+1}^{m+1} A_{1+1} v_{f, j+1}^{m+1}-\left(\dot{\alpha}_{f} \dot{\rho}_{f} U_{f}\right)_{1}^{m+1} A_{1} v_{f, 1}^{m+1}+P\left(\dot{\alpha}_{t} A\right)_{1+1}^{m+1} v_{f, t+1}^{m+1}-P\left(\dot{\alpha}_{f} A\right)_{1}^{m+1} v_{f, 1}^{m+1} \\
& -\left[\frac{h_{f}^{*} V_{L}}{h_{0}^{*}-h_{f}^{*}}\right] H_{10}\left(T_{s}-T_{0}\right)-\left[\frac{h_{0}^{*} V_{L}}{h_{0}^{*}-h_{f}^{*}}\right] H_{f f}\left(T_{s}-T_{f}\right) \\
& +\left[\left(\frac{1+\epsilon}{2}\right) h_{o s}+\left(\frac{1-\epsilon}{2}\right) h_{t s}\right]_{L} \Gamma_{w, L} V_{L}-Q_{w f} V_{L}-D_{i s s} V_{L}-\Gamma_{s} H_{s} V_{L}
\end{aligned}
$$

Examination of Equations 3-31 through 3-35 reveals that each equation, with the exception of 3-34 involves only one unknown vector. The unknown vectors and associated equation set are

$$
\begin{aligned}
& \text { Equation 3-31 }\left(\alpha_{0} \rho_{0} X_{n}\right)^{m+1} \\
& \text { Equation 3-32 }(\alpha \rho)_{a}^{m+1} \\
& \text { Equation 3-33 }(\alpha \rho U)_{0}^{m+1} \\
& \text { Equation 3-34 }(\alpha \rho)_{f}^{m+1} \text { and }(\alpha \rho)_{0}^{m+1} \\
& \text { Equation 3-35 }(\alpha \rho U)_{f}^{m+1}
\end{aligned}
$$

This simplified form results because the new time velocities, $v_{0}^{m+1}$ and $v_{f}^{m+1}$, are known from the first step. Hence, each equation is uncoupled from the others and can be solved independently. Two of the vapor equations have identical coefficient matrices, the vapor density and vapor energy equations. The coefficient matrix is comprised of the known vapor velocities from the first step. This matrix can be inverted once and then multiplied by the appropriate RHS vectors to obtain the $(\alpha \rho)_{0}^{m+1}$ and $(\alpha \rho U)_{0}^{m+1}$ vectors. Equation 3-31, the noncondensible gas density equation has a slightly different form than either the vapor density or energy equations in that the upstream (j) convective terms are replaced with the steady-state initial conditions. As a result, the equations are not coupled and can be solved locally on a node by node basis.

The $(\alpha \rho)_{0}^{m+1}$ elements can be eliminated from the sum density equation, since they were computed using the vapor density equation. This reduces Equation 3-34 to a single unknown vector $(\alpha \rho)_{f}^{m+1}$. It is also a local equation since the upstream terms are eliminated by using the mass flow rate initial conditions. It is solved locally, like the noncondensible density equations, to obtain $(\alpha \rho)_{f}^{m+1}$.

The liquid energy equation is a coupled equation set since each node contains both upstream and downstream terms. A matrix inversion is used to obtain the $(\alpha \rho U)_{f}^{m+1}$ vector.

With the above five dependent variables known, $X_{n}^{m+1}, U_{a}^{m+1}$, and $U_{f}^{m+1}$ are obtained from

$$
X_{n}^{m+1}-\left(\alpha_{0} \rho_{0} X_{n}\right)^{m+1} /(\alpha \rho)_{0}^{m+1}
$$




$$
\begin{aligned}
& U_{0}^{m+1}-(\alpha \rho U)_{c}^{m+1} /(\alpha \rho)_{0}^{m+1} \\
& U_{f}^{m+1}-(\alpha \rho U)_{f}^{m+1} /(\alpha \rho)_{f}^{m+1}
\end{aligned}
$$

The void fraction, $\alpha_{0}^{m+1}$, is obtained from

$$
\alpha_{a}^{m+1}-\frac{\rho_{m}^{m+1}-\rho_{f}^{m+1}}{\hat{\rho}_{0}^{m+1}-\hat{\rho}_{f}^{m+1}}-\frac{(\alpha \rho)_{a}^{m+1}+(\alpha \rho)_{f}^{m+1}-\hat{\rho}_{f}^{m+1}}{\hat{\rho}_{0}^{m+1}-\hat{\rho}_{f}^{m+1}}
$$

where $\rho_{m}^{m+1}$ is the mixture density, $\hat{\rho}_{f}^{m+1}$ is the liquid density, which is calculated form the linearized state relationship using $U_{i}^{m+1}$ and $P^{m+1}$; and $\hat{\rho}_{a}^{m+1}$ is the vapor density, which is calculated from the linearized state relationship using $U_{\mathfrak{o}}^{m+1}$ and $P^{m+1}$.

The linearized state relationships are

$$
\begin{aligned}
& \hat{\rho}_{a}^{m+1}=\rho_{a}^{m}+\frac{\partial \rho_{0}}{\partial U_{a}}\left(U_{a}^{m+1}-U_{0}^{m}\right)+\frac{\partial \rho_{a}}{\partial P}\left(P^{m+1}-P^{m}\right) \\
& \hat{\rho}_{f}^{m+1}=\rho_{f}^{m}+\frac{\partial \rho_{f}}{\partial U_{f}}\left(U_{f}^{m+1}-U_{f}^{m}\right)+\frac{\partial \rho_{f}}{\partial P}\left(P^{m+1}-P^{m}\right)
\end{aligned}
$$

Equations 3-36 through 3-39 define the set of independent variables required by the equation of state.

\subsection{Equation-of-State Relationships}

The equation-of-state relationship in RELAP5 consists of 5 independent variables $\left(P_{v}, U_{0}, U_{f}, \alpha_{0}, X_{n}\right)$. The user via input, may use an alternate set of input, such as $(P, T),(P, X)$, or $(T, X)$. However, that is for user convenience and by completion of the initialization level of RELAP5, all variables are on the same thermodynamic plane. The steady-state balance equations will solve for the variables used in the current transient equation of state. Therefore, no modifications to the equation of state will be required for steady-state initialization.

\subsection{Constitutive Models}

The are several constitutive models required for closure of the momentum and energy equations. These include flow regime maps and models for interphase friction, wall friction, wall heat transfer and interphase mass transfer. A discussion of each of these constitutive models and the special treatment (if any) required for steady-state initialization is presented. 


\subsubsection{Flow Regime Maps}

Four flow regime maps are used in RELAP5, a vertical flow regime map, a horizontal flow regime map, a high mixing volume flow regime map and a ECC mixer map. The vertical flow regime map is the most detailed with nine distinct flow regimes and several transition regimes modeled. The horizontal flow regime map has pre-CHF regimes plus a stratified flow regime. The criteria for selecting and defining transition between flow regimes in the vertical and horizontal flow maps are based on several empirical correlations. Flow regime selection in the high mixing flow regime map is based simply on void fraction. The current flow regime selection logic for the vertical, horizontal and high mixing flow regime maps will not have to be modified for the steady-state initialization.

The ECC mixer volume flow regime map is used in the interfacial heat transfer calculation for condensation. The flow regime selection logic used for this map should be valid for steady-state initialization, however, there should not be any situations in which this map is required for initial conditions in a LWR.

\subsubsection{Interphase Friction}

The interphase drag models in RELAP5 utilize either drift flux relationships or correlations for interphase area and friction factors. In both of these approaches, the relationships are algebraic and will not require any special treatment for steady-state initialization.

There is smoothing between different drag models in the transition flow regimes. This smoothing takes the form of time-dependent exponential smoothing in many cases. In these instances, the time smoothing will be replaced with an appropriate relaxation method for steady-state initialization.

\subsubsection{Coefficient of Virtual Mass}

The coefficient of virtual mass is based on the junction flow regime and is smoothed via junction void fraction between flow regimes. Since there is no time smoothing and the expressions for the coefficient are algebraic expressions, this model will not have to be modified for steady-state initialization. Also, this term drops out of the steady-state equations since it is a coefficient on a time derivative term.

\subsubsection{Wall Friction}

The wall friction calculation requires that the wall surface area wetted by each phase and the appropriate friction factor be determined. The method by which the wall surface area wetted by each phase is determined by the flow regime selected from the flow regime map. As with the interphase drag term, there are not specific wall area and friction factor models for the transition flow regimes. In these instances, a time-dependent exponential smoothing is used between the end flow regimes. 
Therefore, only the transition flow regime methods for computing wall friction will have to be reviewed and modified. For instances where time-dependent smoothing is used, it will be replaced by another method for steady-state initialization.

\subsubsection{Wall Heat Transfer}

Several HTCs are available for selection in RELAP5. The logic to select these correlations is based on several factors such as pressure, wall temperature, noncondensible gas presence, vapor void fraction and CHF. The current logic to select HTCs will not require modification to be used for steady-state initialization. Also, the energy partitioning logic to determine the heat source terms for the phasic energy equations will not be modified for the steady-state initialization.

A steady-state form of the conduction solution will be used to obtain the initial conductor temperature distribution and heat flux. The steady-state conduction solution is described in Section 4.

\subsubsection{Interphase Heat Transfer}

The expressions used for interfacial area and HTCs are selected based on the fluid volume flow regime. As with the interphase drag term, time smoothing is done between correlations in the transition flow regimes. The time smoothing will be replaced with a relaxation method for steadystate initialization.

\subsection{Special Process Models}

Several special process models are present in RELAP5 that simulate processes that cannot be simulated directly by the balance equations and default constitutive models. In many cases, the phenomena modeled by these special purpose models are encountered only in transient situations and would not be present during steady-state operation.

\subsubsection{Choked Flow}

The choked flow model serves as a junction flow boundary condition at the point the flow becomes independent of the downstream conditions. Choked flow would be expected to occur at breaks in the system or at opened safety or relief valves. When choked flow is predicted, an additional equation is solved in conjunction with the upstream momentum equation, therefore, the matrix solution is also modified.

If choking is allowed during the steady-state iterations, logic would have to be added to detect choking and modify the coefficient matrix accordingly. However, since choked flow would not exist in any LWR during normal steady-state operation, choking need not be considered as part of the steady-state solution. 


\subsubsection{Horizontal Stratification and Entrainment}

When horizontal stratification occurs, the junction void fraction may be different than that of the donor volume depending on the junction elevation relative to the stratified level. The horizontal stratification and entrainment model simulates the effects of stratification and vapor or liquid pull through on junction void fraction. As with the choked flow model, the conditions at which this model is activated would not be expected during steady-state operation. Therefore, the horizontal stratification and entrainment model need not be considered during the steady-state iteration process.

\subsubsection{Abrupt Area Change}

Momentum effects of abrupt area changes, from contractions, expansions or orifices are not modeled explicitly by the momentum equations. Therefore, an abrupt area change model is formulated to include these effects. This model will not require any modifications for steady-state initialization and will be included in the iteration process. This model has been used in three of the demonstration problems.

\subsubsection{Crossflow Junction}

Since the momentum equations are based on one-dimensional flow, special process models are used to approximate an improved physical response to a multi-dimensional situation. The momentum flux terms are specially formulated and wall friction is modified for crossflow junctions. The special treatment of these junctions will not require modification for steady-state initialization and will be included as part of the normal steady-state iteration process.

\subsubsection{Water Packing Mitigation}

This special model is required only for the transient solution and the logic for this model will be bypassed for steady-state initialization.

\subsubsection{Counter Current Flow Limitation}

This phenomena will not be encountered during steady-state operation, therefore, the logic dealing with this model will be bypassed during the steady-state iteration process.

\subsection{Component Models}

Several subsystem models are required for complete modeling of LWRs. These include branches, separators, jet mixers, pumps, turbines, valves, and accumulators. This section contains a brief summary of what will be required to include these component models in the steady-state initialization logic. 


\subsubsection{Branch}

The branch component is merely a user convenience to input a series of single volume and junction components. Once past the input processing, the volumes and junctions in a branch are treated in the same manner as other volumes and junctions in the system model. The branch component will not require any modification for steady-state initialization.

\subsubsection{Separator}

The separator model allows the outlet junction void fractions to be a function of the separator void fraction rather than the default model that simply donors the volume void to the junction. The relationships of how the outlet stream void fractions are dependent on the volume void fraction are supplied by the user. One junction void fraction function is required for each outlet junction and they need not be related in any manner.

Even though the junction void fractions are defined from the model, the junction-phase velocities are computed in the standard manner from the phasic momentum equations. Therefore, no modifications to the momentum equation solutions will be required.

The separator model will have to modified to obtain a steady-state energy balance on the separator volume. In steady-state conditions, the energy convection rate into a volume must equal the energy convection rate out of the volume. Since in the current formulation, the void fraction of the two outlet junctions are based on user supplied correlations, there is no method to ensure a volume energy balance.

In order to ensure that the separator volume steady-state energy equations are balanced, the steadystate initialization process will have to adjust one of the outlet junction void fractions. Since during normal separator performance the vapor outlet junction void fraction will be close to 1.0 , the void fraction of the liquid fall back junction will be adjusted. The user-supplied void fraction function for the vapor outlet junction will be used and the separator void fraction or the user-supplied function will be adjusted to achieve the liquid fall back junction void fraction required for a separator energy balance.

\subsubsection{Jet Mixer}

Momentum exchange between two fluid stream entering a control volume at different velocities is not modeled by the standard RELAP5 momentum equations. Fluid stream momentum exchange is required to model BWR jet pumps and may be needed at ECC injection points. The jet mixer model simulates stream mixing by computing another differential pressure term for the momentum equations of the mixing streams (junctions).

The logic for this optionally activated model is already part of the momentum equation formulation and will not require modification for steady-state initialization. 


\subsubsection{Centrifugal Pump Model}

The pump model is a component model requiring a single hydraulic volume and two flow junctions. The pump model provides a head term that is equally distributed between the inlet and outlet junction momentum equations and a dissipation term that is included as a energy source term in the pump volume energy equation. The pump head and energy dissipation is a function of pump speed, pump volume volumetric flow and volume density.

The energy dissipation term will automatically be included in the steady-state initialization scheme through the volume energy equation and in the overall energy balance. The two parameters that may require special consideration for steady-state initialization are the pump head and initial pump speed.

The pump head will be directly included in the inlet and outlet junction momentum equations as part of the iteration process and the existing RELAP5 coding will be used for this. The problem with the pump head and steady-state initialization arises from the fact that the pressure losses around the system must be balanced by the pressure increase across the pump. There are two optional methods that will be implemented to ensure this. The first option is to have steady-state initialization adjust the pump speed to balance the system pressure drop. The second option is to have the initialization process adjust a junction loss coefficient in the loop (preferably at the pump outlet) to make up for the pressure imbalance. The second option is valid only if the developed pump head is greater than the system pressure drop. The new initial pump speed or computed loss coefficient computed by steady-state initialization will be saved and used in the transient calculation.

There are several options for changing the pump speed during the transient. These options include tripping the pump or allowing the pump impeller speed to change based on a shaft torque balance. Any pump angular speed changes are computed by integrating a pump speed equation. During the steady-state iteration process, the pump speed equation will be bypassed.

\subsubsection{Turbine Model}

The turbine model modifies the form of the momentum equations and energy equations. If this model is to be included in steady-state initialization, a normalization procedure will have to be developed to ensure the turbine removes the correct amount of initial energy. For the purposes of the next phase of steady-state initialization, it is recommended that balance-of-plant models (such as the turbine model) not be included.

\subsubsection{Valves}

Several valve model options are available for use, however, they all serve the same purpose: they define the junction flow area. The valve model can obtain flow areas from opening and closing tables, instantaneous open and close trips and from the control system. As far as steady-state initialization is concerned, the junction flow area should not change during the iteration process and 
the area used in the steady-state initialization calculation must be consistent with that defined by the control system or area tables.

The initial junction area used for steady-state initialization must be obtained from the valve models rather than relying of the area input on the junction data cards. Therefore, the valve models will be evaluated prior to any steady-state iteration to ensure the junction area used will be consistent with that used when the transition to the transient module is made.

\subsubsection{Accumulator}

The accumulator is isolated from the rest of the system during normal operation. It is only during transient conditions that the accumulator influences the system response. Therefore, no special treatment for the accumulator will be required during steady-state initialization.

\subsubsection{ECC Mixer Model}

The ECC mixer is a model that will only become active in the transient once ECCS injection begins. Since no ECC injection would be expected during normal operation, this model will not require an interface with the steady-state initialization logic. 


\section{HEAT STRUCTURE}

Heat structures such as fuel rods, piping vessel walls, and internal structure can be modeled in RELAP5. The location of the heat structures and the material(s) the heat structures are composed of is specified by the user. The structures can have an internal heat source (core conductors) and can transfer energy to or between volumes. Currently, the user can have the code compute the internal node temperature distribution or the user can input the initial node temperatures. For steady-state initialization, the code must compute the initial internal node temperatures in order to ensure a conductor heat balance. Therefore, the option of the user specifying the initial node temperatures will be flagged as an error if the steady-state initialization option is used.

There are several heat structure configurations that can be modeled and, therefore, must be considered for proper initialization of heat structures. For all configurations, the internal node temperature must be adjusted to achieve the desired initial conditions. These configurations and the pertinent considerations are

- for all powered heat structures, the internal heat generation rate must equal the heat transfer rate through the conductor surface(s)

- for passive (nonpowered) heat structures that have a fluid volume on one side and are insulated on the other side, the initial heat transfer rate must be zero

- for passive heat structures that have fluid volumes on both sides or a fluid volume on one side and a boundary condition on the other side, the initial heat transfer rate in one side of the conductor must equal the heat transfer rate out the other side

There is currently a subroutine in RELAP5 that provides the initial heat structure node temperatures by solving the steady-state conduction equations and satisfying the above constraints. In the current version of RELAP5 this subroutine is called only during initialization to provide an initial guess for conductor temperatures. After the initial call, the transient conduction equations are solved while running a transient to obtain steady-state conditions.

During this transient steady-state solution, certain variables are constrained, such as system power, to the time $=0.0$ value. To overcome thermal time constants that may be large, the code artificially reduces the $\rho C_{p}(T)$ term to a small number for each advancement. For the heat structures, the transient form of the heat conduction equations are therefore computed. The heat conduction solution procedure is shown schematically in Figure 4.1, where the call to subroutine HTADV is made from subroutine TRAN for each time advancement. When the steady-state criteria is satisfied, the heat conductors will be initialized within some degree of accuracy of the true steady-state values.

As stated earlier, a steady-state conduction solution is available in RELAP5 (subroutine HT1SST). This subroutine was successfully incorporated into the steady-state iteration logic for one of the demonstration problems to illustrate the feasibility of using the existing steady-state heat conduction 


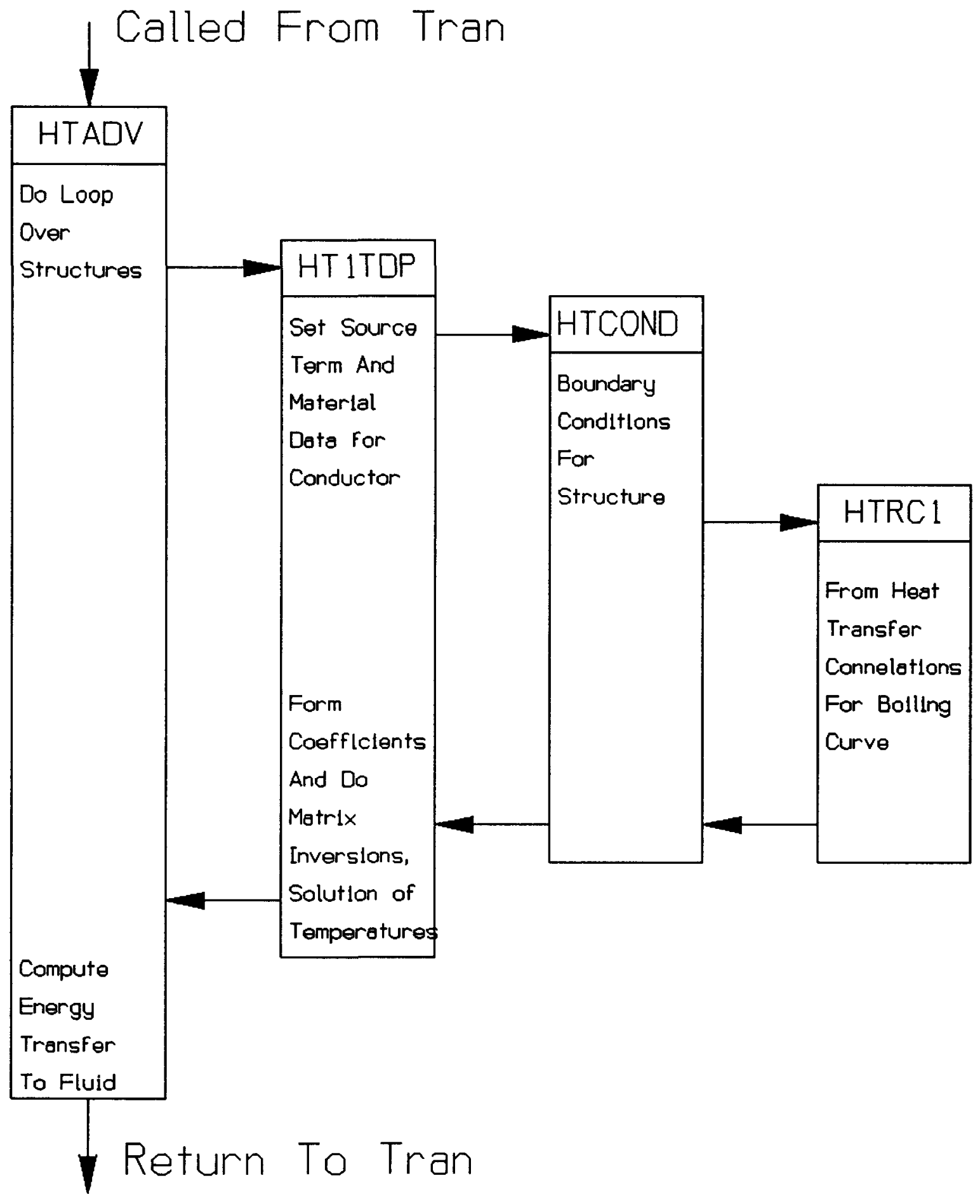

Figure 4.1 RELAP5 conductor solution procedure 
solution. The transient and steady-state forms of the conduction equations are discussed in the next sections on method.

\subsection{Conduction Equation and Finite Differencing - Transient Form}

The conduction equation, its finite differencing, and solution are discussed in Reference 1 . The equations are briefly reviewed in order to relate them to the steady-state solution. The RELAP5 code solves the one-dimensional heat conduction equation given in its integral form

$\int_{V} C_{V}(T, x) \frac{\partial T(x, t)}{\partial t} d V=\int_{S} k(T, x) \nabla T(x, t) \cdot d S+\int_{V} S(x, t) d V$

where

$\mathrm{C}_{\mathrm{v}}=$ volumetric heat capacity, $\mathrm{J} / \mathrm{m}^{3}-\mathrm{K}$

$\mathrm{T}=$ temperature, $\mathrm{K}$

$\mathrm{V}=$ conductor volume, $\mathrm{m}^{3}$

$k=$ thermal conductivity, $w / m-k$

$s=$ conductor surface area, $\mathrm{m}^{2}$

$S(x, t)=$ volumetric heat source, $w / m^{3}$

with boundary conditions given by the expression

$A(T) T(t)+B(T) \frac{\partial T(t)}{\partial n}=D(T)$

or

$-\frac{\partial T(t)}{\partial n}=\frac{A(T) T(t)-D(T)}{B(T)}$

where

$\mathrm{A}(\mathrm{T})=$ temperature boundary condition, $\mathrm{K}$

$B(T)=$ conductivity, $w / \mathrm{m}^{2} \mathrm{~K}$

$D(T)=$ heat flux boundary condition, $w / \mathrm{m}^{2}$

For the numerical solution, Equations 4-1 and 4-2 are first finite differenced, and then weighting factors are applied. These weighting factors allow for the differenced equations to range between a fully implicit differencing $(\omega=1.0)$, to a fully explicit differencing $(\omega=0.0)$, as

$g_{m}\left(\frac{T_{m}^{n+1}-T_{m}^{n}}{\Delta t}\right)-\omega \delta_{m}^{n+1}+(1.0-\omega) \delta_{m}^{n}$ 
where $\delta_{m}$ is the finite differenced form of the RHS of Equation 4-1 above, for mesh node $m$ and $g_{m}=C_{v} V$. (Note: for boundary condition, $\omega$ is always set to 1.0, or fully implicit.) By defining $\omega=1 /(1+\sigma)$, and multiplying the LHS of Equation 4-1 by $\sigma$, the formulation can be used for expressing both the transient and steady-state form of the conduction equation, as formulated in RELAP5, as

$$
\sigma\left(\frac{T_{m}^{n+1}-T_{m}^{n}}{\Delta T}\right)=\frac{\delta_{m}^{n+1}}{1+\sigma}+\frac{\sigma \delta_{m}^{n}}{1+\sigma}
$$

For the transient expression, use $\sigma=1$, which defines $\omega=1 / 2$, which is the Crank-Nicholson implicit formulation. For the steady-state expression, set $\sigma=0, \Delta t=1.0$.

When the RHS of Equation 4-5 is expanded (differencing Equations 4-1 and 4-2) and all terms collected, the finite difference form of the conduction equation for mesh node $m$ is

$$
a_{m} T_{m-1}^{n+1}+b_{m} T_{m}^{n+1}+C_{m} T_{m+1}^{n+1}=d_{m}
$$

For a given geometry, divided into $m$ mesh points ( $m-1$ regions) an $m \times m$ tridiagonal matrix in the coefficients $a_{m}, b_{m}, c_{m}$ is generated, as well as a coefficient vector in $d_{m}$. The solution of the equations can then be obtained by Gauss elimination.

\subsection{Steady-State Procedure}

From necessity, the procedure for the solution of the steady-state conduction equation, when using convective fluid dependent flux boundary conditions is very similar to the transient solution.

However, for steady state, the field equations must be brought into balance without advancing time and with temporal derivatives equal to zero. The conduction equations at steady state are much simpler than the transient form, free of time constants, and should converge much more quickly.

For the steady-state solution, the present RELAP5 subroutine HT1SST will be used with only minor modification. For calls to HT1SST, the time $=0$ values of the source term $\left(\int_{v} S d V\right.$ of Eq. 4-1) must be available. Also for constant or time-dependent boundary conditions, the time $=0.0$ values must be known. Then a steady-state conduction solution can be made. However, for convective boundary conditions, the surface flux is a variant, in terms of the fluid conditions, and the conduction solution becomes an iterative procedure with the hydrodynamics and fluid state equations.

The transient conduction subroutine HT1TDP could also be modified to provide a steady-state solution. However, this looks like a more difficult approach, as it appears that the weight factor $\omega$ is built into the code $(\omega=0.5)$, and many lines of coding might have to be modified. Also, setting $\Delta T=0.0$ might cause problems. 
The transient (HT1TDP) and steady-state (HT1SST) subroutines are now compared, in order to evaluate the capability of HT1SST to provide for the steady-state solution and initialize variables. This comparison is shown in Figure 4.2. From Figure 4.2, it is seen that HT1SST evaluates the source terms and boundary conditions at $t=0.0$, and provides the temperature solution. It does not, however, do rupture and strain calculations. These calculations should not be required for steady state. Thus, subroutine HT1SST can be used for the heat structure steady-state conduction solution, with minor modifications.

As discussed in the demonstration problems (Section 10), the suggested procedure for steady-state heat conduction was tested. A steady-state heat conduction driver SSHTAV was written (cloned from HTADV). It then calls subroutine HT1SST. The procedure works well and may be used to obtain the steady-state heat conduction solution. 


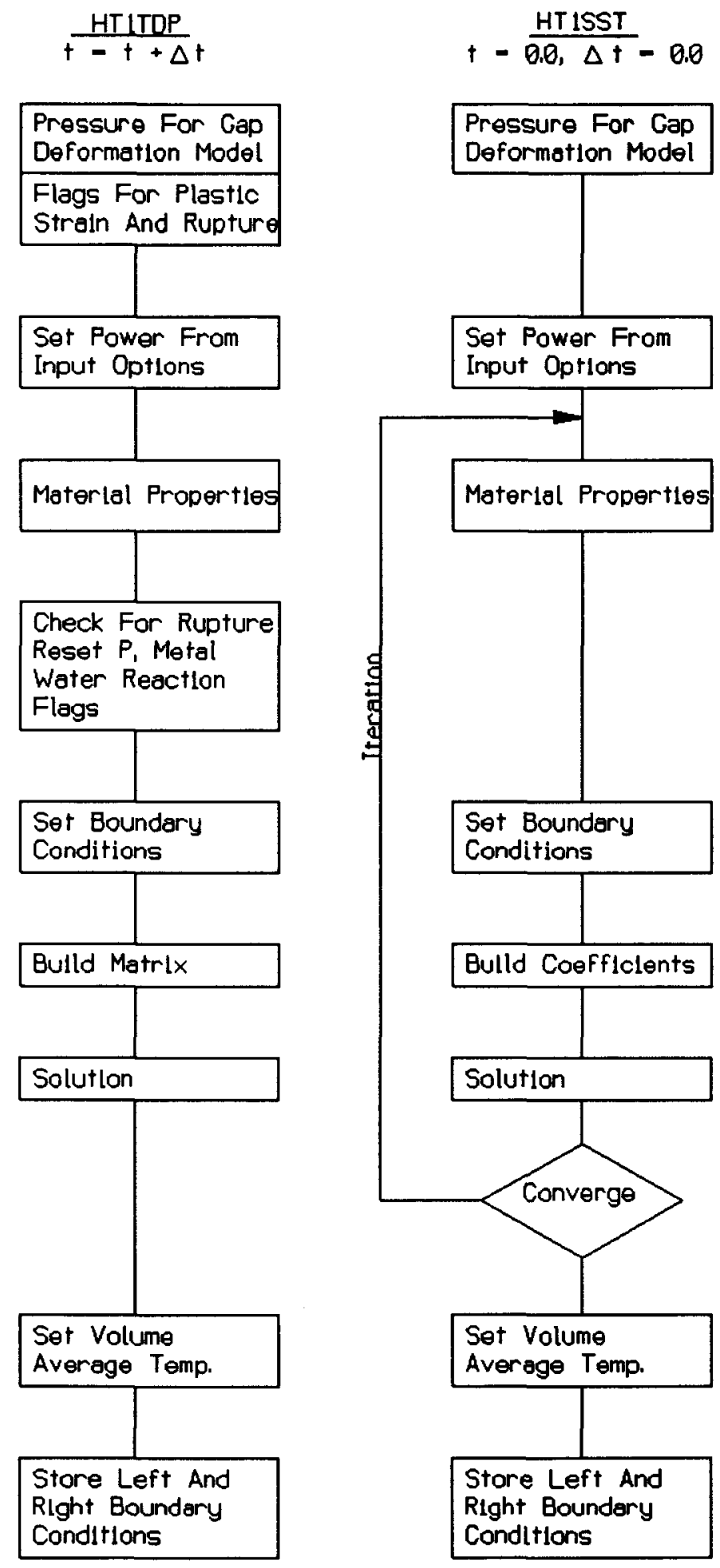

Figure 4.2 Comparison of RELAP5 transient and steady-state conduction subroutines 


\section{CONTROL SYSTEM}

The control system models in RELAP5 allow the user to simulate typical control systems (i.e., feedwater control, turbine valve modulation, bypass valves) in the plant during a simulated transient. The control system models can also be used to evaluate any arbitrary algorithm using RELAP5 computed quantities. The output of these control systems can be monitored by a trip, edited, or used to define characteristics of components within the system such as valve area, pump speed, junction flow, etc.

The control system models currently available in RELAP5 have two type of uses. The first is the "standard" use, in which the user constructs a control system by interconnecting the control blocks in a logical manner to define some plant controller or perform a side calculation not performed by the code. The second use is through a generic set of controllers, called "self-initialization" controllers, used to force the transient solution to maintain the input value of a few parameters (such as cold leg temperature, loop flow, pump speed and secondary flow or pressure).

The self-initialization controllers will not be required for the new steady-state initialization method. With steady-state initialization, the input quantities the user wants to maintain (such as cold leg temperature, secondary pressure, etc.) will be clearly identified and will remain at those input values through the steady-state iteration process. Since the transient solution will not have to be run to achieve steady-state conditions and the initiating events can occur immediately after steady-state initialization has converged, these self-initialization controllers will no longer be needed. Logic will have to be incorporated in the input processing to ensure that if the steady-state initialization option is activated, an error message will be written if self-initialization controllers are also used.

The "standard" control system can be used to define a parameter that is used in a constitutive or component model. Because of this, control system defined quantities may be part of the steadystate solution of overall balance equations. There are two aspects to address for control systems, first is steady-state initialization of the control systems themselves and second, steady-state initialization of components and/or constitutive model that have parameters defined by the control system.

For initialization of the control system itself, one of two approaches can be used. The first and simplest is not to make the control system calculation part of the steady-state iteration process. In this case, the initial control block values supplied for the user will be used to define the controlled component or constitutive model parameters initial values. These parameters will remain constant throughout the steady-state initialization process. Once a converged steady-state thermal-hydraulic solution has been obtained, all the parameters required as input to the control system will have been updated to reflect the computed steady-state conditions and the control block outputs will then be computed. The current control block solution is simply a sequential treatment of one block at a time and may not be in the same order in which the control blocks are connected. Therefore, an iterative solution will have to be developed to make several passes through the control block calculations to ensure converged block initial conditions. The newly computed initial control block values will be compared to the input initial values and where differences exist larger than a preprescribed convergence criteria, a warning message will be written. Both the input and computed values will 
be edited so the user can clearly see any differences. The advantage to this method is that it is the easiest to implement and has proven to work adequately in the RETRAN initialization package (Refs. 2 and 3). The disadvantage is that is incumbent on the user to revise the input control block initial conditions if a discrepancy occurs.

The second approach is to compute new control block outputs during each steady-state iteration. The advantage of this approach is that after a converged steady state has been obtained, both the control system and hydraulics will be completely consistent. The user will not be required to revise the control block initial conditions to ensure consistency. The disadvantage to this approach is that it can lend instabilities to the rate of steady-state convergence since the control system logic may use thermal-hydraulic information and the control system output may have direct impact on the same computed thermal-hydraulic information. In some instances, a feedback loop may develop that would not permit a steady-state solution. In addition, it will still be advisable for the user to review the input and computed control block initial conditions to ensure the discrepancies are acceptable.

The second aspect to discuss is the steady-state initialization of components and/or constitutive models that have parameters defined by the control system. All the control blocks are evaluated within one subroutine and the control block output stored. If a component parameter is defined by the control system, that parameter is defined when the calculation for the component is done. In other words, nothing will have to be done for specific component or constitutive models as far the control system is concerned since the logic is already built in those models for the control system. 


\section{TRIP SYSTEM}

Only elapsed time trip signals (those that trip at time zero within no delay) will be allowed during the steady-state solution. This will be done to prevent nonconvergent oscillations that may be introduced by pressure and temperature trips being activated by early and possibly poor initial estimates of the trip signal parameters. 



\section{REACTOR KINETICS}

A point kinetics model is optionally available in RELAP5 to define normalized power. The power distribution is defined by the user initially and remains constant throughout the transient. Reactivity feedback can be modeled through a series of tables of reactivity versus moderator density and/or fuel temperature. In addition, reactivity can be contributed by any arbitrary function through the control system. A scram table can also be supplied to model control rod movement.

By definition, during steady-state operation, the net reactivity must be zero and the power should be time invariant. Currently, RELAP5 is run with null reactivity feedback quantities to maintain constant initial power in order to achieve steady-state conditions. Once the desired steady-state results are obtained by running the transient solution, the user must restart and supply the actual reactivity feedback tables along with the change cards to initiate the transient.

The initial power must also remain constant during the steady-state iterations. Therefore, the point kinetics equations will not be evaluated during the steady-state iteration process. However, after a converged set of steady-state initial thermal-hydraulic conditions have been obtained, the initial reactivity will be evaluated. This initial reactivity (both from feedback terms and explicit reactivity) will be used to bias the reactivity at each time step in the transient so the net reactivity is measured as a change from time zero. In order to evaluate the initial bias reactivity, a subroutine will have to be written to compute and store the initial contribution from each reactivity component. The new subroutine will be called from the steady-state initialization driver after the thermal-hydraulic conditions have converged thus ensuring consistency between the steady-state thermal- hydraulic conditions and the reference feedback reactivities. 



\section{OVERALL SYSTEM ENERGY BALANCE}

Various models are available in the RELAP5 code to model energy addition or removal from control volumes. The typical sources of energy are generally the core (modeled by the use of the heat structures with internal power generation) and the pumps. In addition, local source terms in the volume energy equations due to friction are computed. In PWRs, the energy removal is often treated using heat conductors with fluid volumes on both sides as a heat exchanger. In BWRs the energy is removed by an open flow system that consists of boundary conditions for feedwater inlet and steam outlet. Small amounts of energy can also be removed by the makeup/letdown system or from environmental heat losses. On a local basis the steady-state energy equations will be solved for each volume, however, for a consistent set of steady-state conditions, the energy removal and addition rates must be equal for each closed flowing network. In order to ensure this, special treatment of certain models will have to be devised which is the topic of this section.

\subsection{Flow Network Identification}

For steady-state conditions within a closed flowing network or system in RELAP5 terminology, the energy addition and removal rates must be equal. A flowing network is defined as a set of volumes that are interconnected by active flow paths. As an example, a PWR primary side is a closed flowing network. Any branches off of the primary that are connected by a closed valve are considered a separate network. The steam generator secondary and primary are connected via heat conductors but not flow paths, therefore, the steam generator secondary side and associated steam line volumes are considered a separate flow network. Each flow network must be identified to ensure that the energy sources and sinks are equal for that network. In most instances, some adjustments will have to be made to obtain an energy balance.

The RELAP5 user has a great deal of flexibility on nodalization, component numbering, and placement of heat sources and sinks. Logic will have to be incorporated into the code to identify the energy sources and sinks that belong to each network. This information will then be used by the steady-state initialization logic to sum all the flow network sources and sinks and make the appropriate adjustments for an overall energy balance. The specific adjustments are described in the next two subsections.

\subsection{PWR System Energy Balance}

Typical PWR models contain two active flow networks, the RCS and the steam generator secondary side. The RCS is made up of the reactor core, the vessel, the cold and hot legs and steam generator tubes. RCS energy sources generally include the reactor core and the RCPs. The initial core power is considered constant for steady-state initialization with the energy transferred to the coolant from the core conductors equals the initial power. The energy added by the RCPs is a function of the initial pump speed, the flow through the pump and the fluid density. Also, the effects of friction are included in the volume energy equations. 
The majority of the energy added to RCS is removed by the steam generator. A smaller amount of energy may also be removed from the system by environmental heat loss or through the letdown/makeup system.

\subsubsection{Steam Generators}

Steam generator tubes are modeled with two-surface conductors with fluid volumes on each side. The energy transfer rate through each conductor is determined by the adjacent volume fluid temperatures and surface heat transfer coefficients. The required energy transfer rate through the steam generators in order to obtain a RCS energy balance is a boundary condition that can be computed. However, the sum of the energy transfer rates through all the heat conductors that makeup the steam generators will not necessarily match the desired boundary condition. Therefore, the steady-state initialization process will have to make adjustments to ensure overall system energy balance. Two adjustments will be required, one to get the correct energy transfer rate from the RCS and the other to get the energy transferred to the steam generator secondary removed.

The power added from the core conductors is a fixed value during steady-state initialization. Other quantities such as the pump heat and environmental loss (which are a function of RCS volume properties) are refined with each steady-state iteration. With these quantities defined, the required net energy transfer rate through the steam generators can be computed and compared with the steam generator heat transfer rates computed by RELAP5. In all probability the desired and computed heat transfer rates will not match. Generally, the initial RCS flow rates and leg temperatures are known and the user will desire to maintain those input values. Therefore, the secondary pressure will be adjusted to get the correct steam generator heat transfer rates. Since the majority of the secondary fluid is at saturation temperature, the pressure adjustment will have a direct effect on secondary temperature and consequently heat transfer rates. If more than one steam generator is modeled, the user will have to specify the fraction of power to be removed by each steam generator.

The energy transferred from the RCS to the secondary must also be removed. For system transient analysis, it is not practical to model the complete balance of plant. Consequently, secondary energy removal is generally modeled with an open flow system consisting of boundary conditions. The feedwater is typically modeled with a time-dependent junction downstream of a time-dependent volume where the fluid conditions are specified. The steam line is generally terminated at a pressure boundary defined by a time-dependent volume. In order to have an overall secondary energy balance, Equation 8-1 must be satisfied.

$Q_{s 0}=\left(W^{*} h\right)_{\text {steam }}-\left(W^{*} h\right)_{f w}$

where

$\mathrm{O}_{\mathrm{sg}}=$ energy transferred to the steam generator, $\mathrm{J} / \mathrm{s}$

$\mathrm{W}=$ feedwater or steam flow, $\mathrm{kg} / \mathrm{s}$

$\mathrm{h}=$ flowing enthalpy of the fluid, J,kg 
Equation 8-1 will be rearranged to solve for a the change in feedwater enthalpy (enthalpy bias) required for the energy balance as shown in Equation 8-2.

$h_{\text {bias }}=h_{\text {steam }}-h_{\mathrm{fw}}-Q_{\mathrm{sq}} / W$

The enthalpy bias computed from Equation 8-2 will be used as an offset to the input time-dependent volume conditions upstream of the feedwater junction. 



\section{IMPLEMENTATION PLAN}

This section describes how steady-state initialization will be implemented in the current RELAP5 framework. The general approach is to provide the RELAP5 user with the new steady-state initialization option with a minimum impact on their current input model. In other words, the user will be able to use RELAP5 in the same manner as currently available without input deck modifications. If the user wants to activate the steady-state initialization solution, a minimal set of additional input cards will be required and the base RELAP5 input will not have to be modified. This approach will minimize the effort required for the code user to begin using the modified version of RELAP5.

Steady-state initialization will be an option that is user requested and will require that several new subroutines be written and interfaced with the rest of the code. New subroutines will be written for input processing, the steady-state iterations and for editing parameters adjusted by steady-state initialization. Flow diagrams of how the steady-state initialization option will be interfaced with RELAP5 are described in Section 9.1. Section 9.2 describes the input requirements for the steadystate option, specifically including modifications to the base deck and the new input.

Major revisions to a code such as RELAP5 require some mechanism to track and document those revisions so they can be incorporated into the mainstream versions through the normal version control processes. Section 9.3 describes the process by which the modifications for steady-state initialization will be maintained.

\subsection{Flow Diagrams}

After reviewing the RELAP5 solution procedures and developing a testbed code, an overall organization for implementing a steady-state solution has been developed and is given in this section. The intent is to have minimal impact on the code (not a lot of updates to current subroutines). The implementation will effect the read/initialization and transient portions of the code.

\subsubsection{Input - Initialization Modifications}

A control flag will be required to identify the steady-state solution option. Presently, problem control is input on Card 100, "Problem Type and Option". Word 2 on this card, when Word 1 is "NEW", has two options. These are the transient steady-state option STDYST and transient option TRANST. Word 2 options should be expanded to include a third option, SSTATE for the steady state. This control word can then be used to set variable IROUTE, which controls program flow as shown in Figure 9.2. The control word can also be used to specify the processing of any new input required for true steady-state solution. 
The main driver program RELAP5 calls input driver subroutine INPUTD. For a "NEW" problem, various read routines are called from subroutine RNEWP to process various system data and all component data. As shown in Figure 9.1, a new subroutine RSSTAT can be added to read data required for the steady-state initialization option (pressures at certain volumes, loop flows, junctions where loss coefficients are to be specified, etc.). The diagram of Figure 9.1 is for problem type "NEW" only.

After all data is read, the initialization level is called. At this level, all controls, components, etc., are initialized (and checked) from the input data. Again, a new subroutine can be added which will build a file that will include data that specify junctions where loss coefficients are to be computed, which volume pressure are fixed, etc.

\subsubsection{Program Control}

The driver program RELAP5 provides overall program control. This routine will be modified, as shown in Figure 9.2 to include control of the steady-state solution option. This will be done using the control word "IROUTE" which will be set to six from the input problem option (Word 2, Card 100) SSTATE.

\subsubsection{Iteration Control}

The proposed iteration control is shown in Figure 9.3. The flow diagram shows the analogy between the transient and steady state. The diagram for the transient is a general diagram of the transient control subroutine TRAN. Subroutine SSADVC was written to control the steady-state iterations. This subroutine will control the steady-state solution and the similarities to the transient flow can be seen. A new subroutine for steady-state heat transfer (SSHTAV) does the same functions as the transient subroutine (HTADV). The loop called by HYDRO is within the steadystate iteration as shown. The transient subroutines VIMPLT, PIMPLT, and SIMPLT are replaced by similar steady-state subroutines SSVIMP, SSPIMP, and SSSIMP. These subroutines can be included/excluded in the source code using DEFINE statements.

\subsection{Input Modifications}

As stated earlier, it is desirable to impact the typical RELAP5 user as little as possible with the incorporation of steady-state initialization in the code. The user should be able to use the code in the same way that is currently available with the same input model. Therefore, steady-state initialization will be incorporated as an option and if that option is not used, no modifications of the standard RELAP5 input will be required. If the option is used, an additional set of input data will be required to define where volume thermodynamic parameters and junction loss coefficients are to be fixed, what types of energy removal systems are modeled and the desired removal rates. These input changes are described in the following sections. 


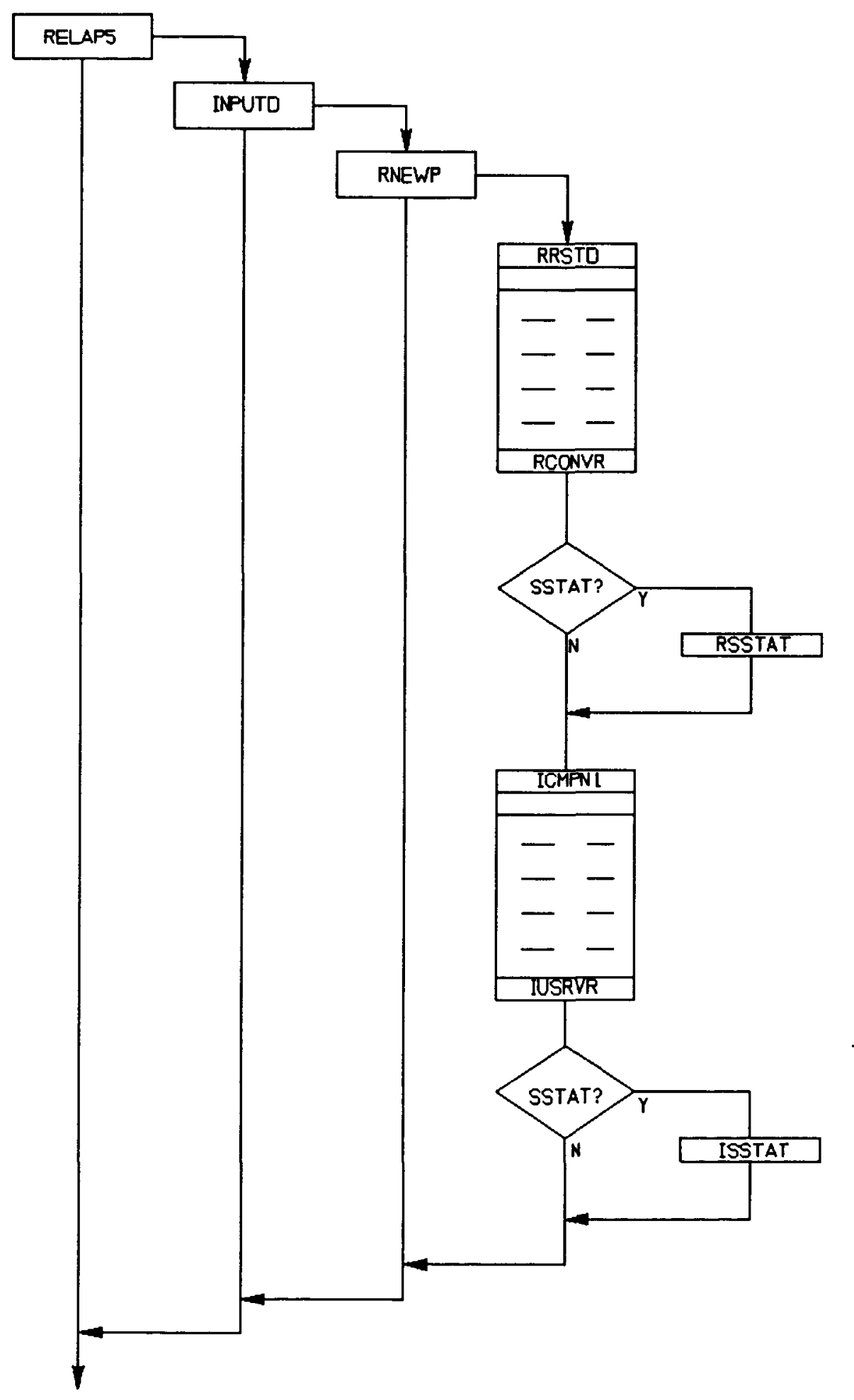

Figure 9.1 Partial flow diagram for steady-state read and initialization 


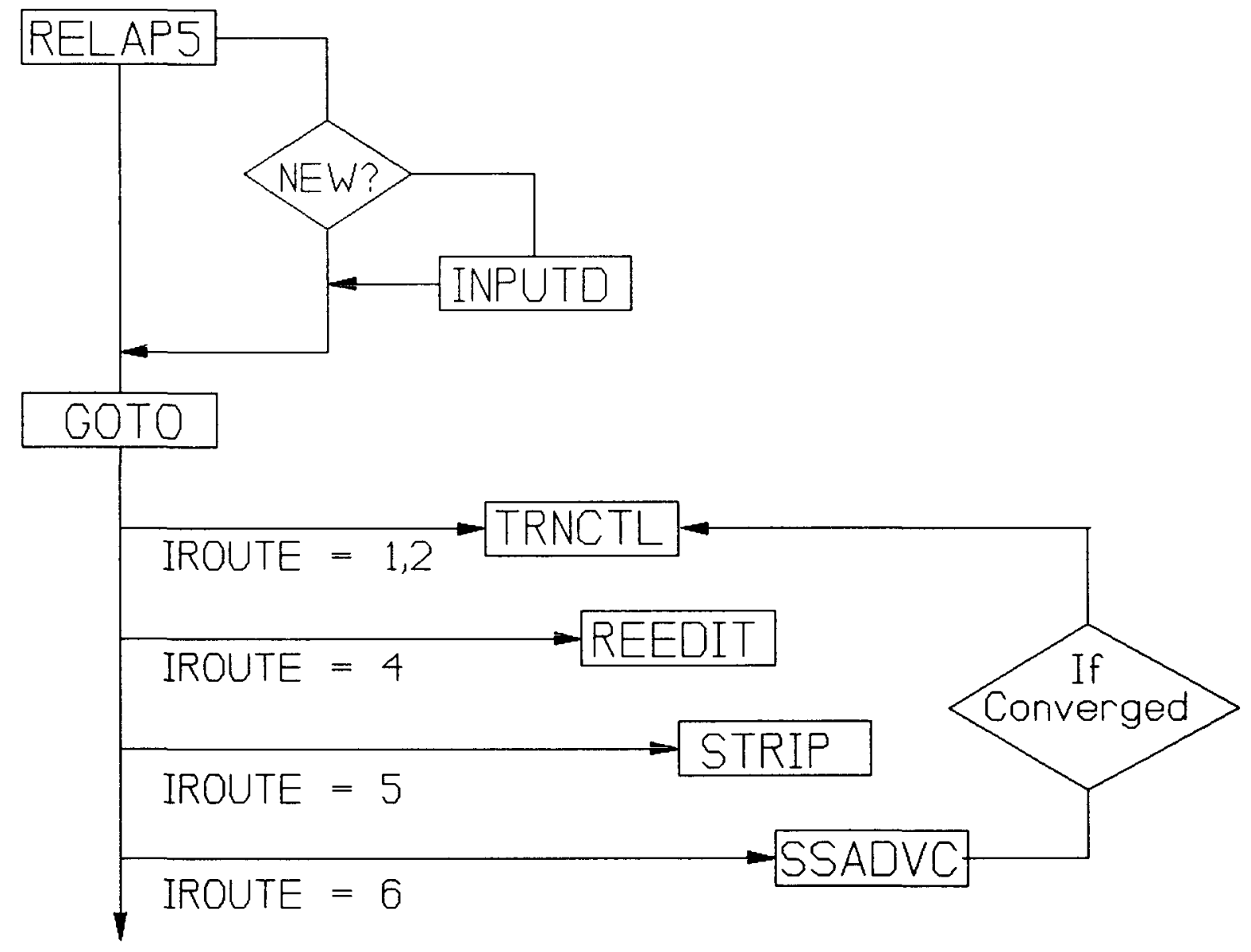

Figure 9.2 Partial diagram for program control 
RELAPS

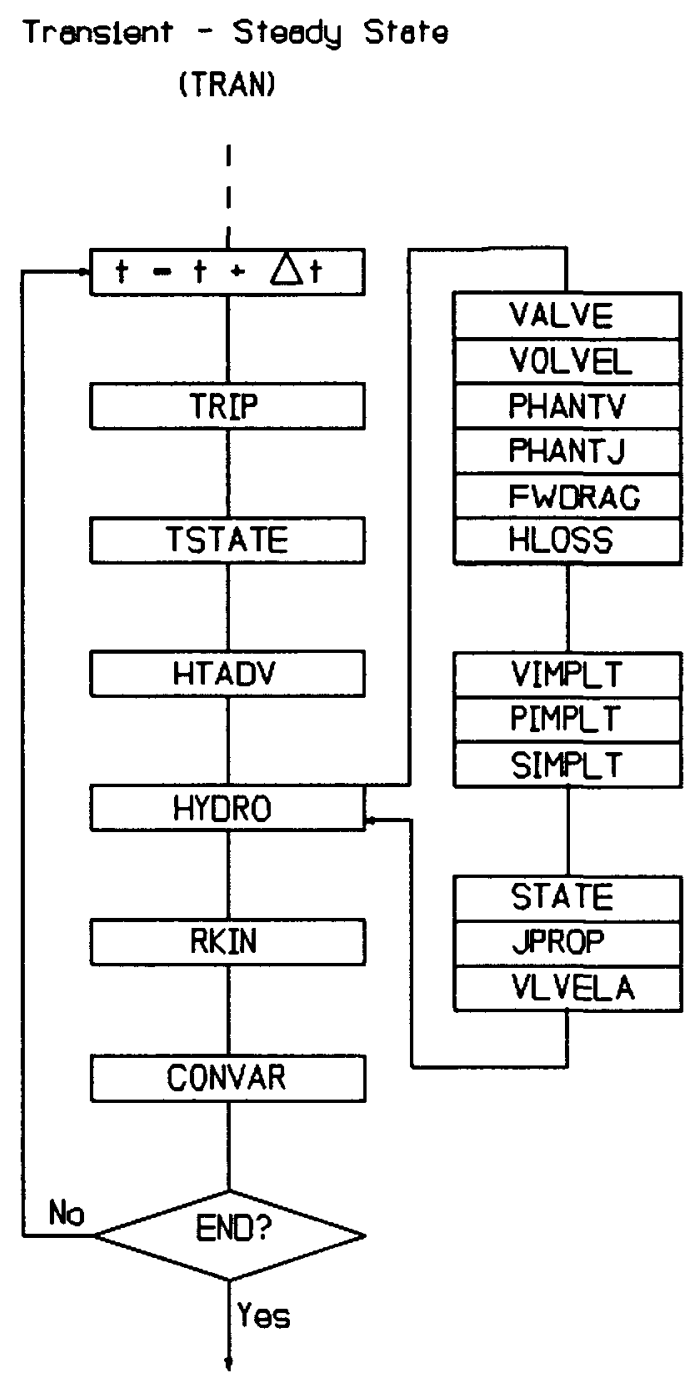

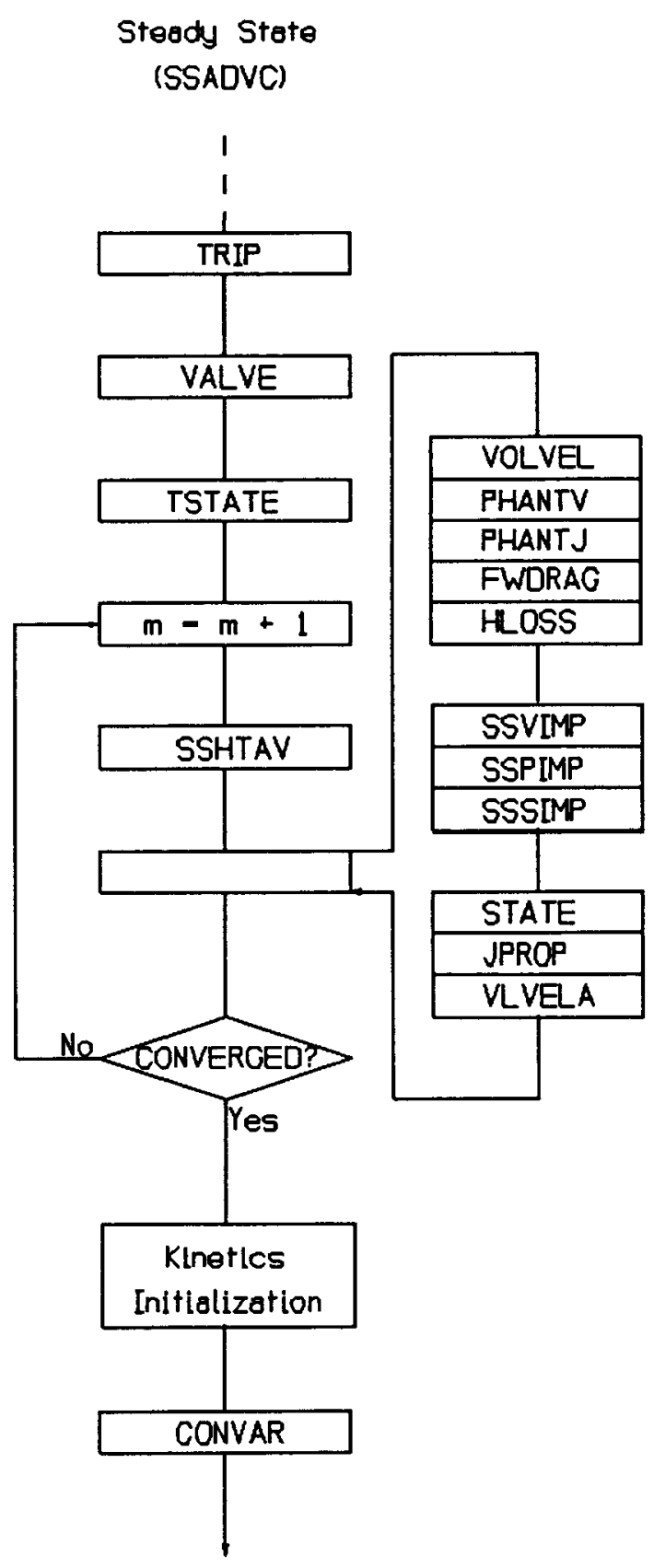

Figure 9.3 Transient and steady-state advancement schemes 


\subsubsection{Initial Volume Thermal-Hydraulic Conditions}

Currently the user specifies initial thermodynamic state for each volume. In theory, the new steadystate initialization would need thermodynamic information only in the volumes in which the properties were to remain fixed during initialization. However, if the volume input requirements were to be modified, users would have to modify their base input models to use the steady-state option instead of supplying a small amount of additional information. A second motive for maintaining the current volume input is that it will provide initial guesses for all volume properties to start the steady-state iteration process.

To define where the volume thermodynamic properties are to remain fixed during steady-state initialization, two additional input cards will be required. One input card will list the volume numbers for which the pressure shall remained fixed, and the other data card will list the volumes for which the input internal energy will remain fixed. The pressure and internal energy will not have to be fixed in the same location. The initial information for these volumes will be maintained during the steady-state iterations. The initial conditions supplied for the other volumes will be replaced with those computed by steady state.

The initial guesses for volume thermodynamic conditions (supplied through the normal input) will not have to be accurate except for those which will remain fixed. As an example, the pressure for all volumes on a PWR primary can be input as the same value assuming only one pressure will remain fixed. Therefore, even though the initial conditions for all volume must still be supplied, it will only require crude guesses and minimal effort on the user's part.

\subsubsection{Junction Loss Coefficients and Flow}

The junction loss coefficients are currently supplied for all junctions. With the new steady-state initialization, one or more of the junction loss coefficients can be computed to balance the junction momentum equations. If the steady-state initialization is used, the junction numbers for which loss coefficients are to be computed will be listed on a new data card. For the junctions where the loss coefficients are to be computed by steady-state initialization, the input loss coefficient can be any arbitrary value since it will be recomputed.

A minimum number of junction flows must be specified with steady-state initialization. The user must define the flow rate in one junction per loop and at flow splits in parallel channels, tees and manifolds. The rest of the junction flow rates can be supplied as zero and the sum continuity equation will be used to propagate the known flow rates around the system. No new input will be required for this option and the junction flow rates can still be supplied as they are in the current input. The user will have the option of supplying all the junction flow rates (as currently required) or the minimum set of flow rates required to propagate the flow to other junctions.

Optionally, a method could be developed to allow the flow rate through a channel or several parallel channels to be computed given a $\Delta P$ across the channel. If this method is made available, an additional input data card would be required to specify the volume numbers bounding the channel(s) 
and the junction numbers with the channel(s). The pressure in the bounding volume would have to be input and fixed for the steady-state iteration process.

\subsubsection{Overall Energy Balance Specification}

In order to ensure the power sources and sinks within a flow network are equal, some additional information identifying the sources or sinks will be required. For PWR models, a data card for each steam generator will have to be supplied where the input will consist of the fraction of power to be removed by the steam generator, a list of conductor numbers that are used to model the tube walls, the volume in which the secondary side pressure is fixed and the steam generator feedwater junction and steam line junction numbers. From this information, steady-state initialization will be able to adjust the secondary pressure to get the correct energy transfer rates and to adjust the feedwater enthalpy to get an energy balance on the secondary side.

For BWR models only the feedwater and steam line junction numbers will need to be identified so the feedwater enthalpy can be adjusted to obtain a reactor vessel energy balance.

For both PWR and BWR models, a data card will have to be supplied to identify conductor numbers that simulate environment losses so these conductors can be factored into the overall energy balance.

\subsubsection{Steady-State Convergence Criteria}

The convergence of each steady-state balance equations will be tested at the end of each iteration by comparing against predefined convergence criteria. Default values for the convergence criteria will be defined and used unless the user chooses to over-ride one or all of the default criteria by supplying an optional data card.

\subsection{Maintaining Code Modifications}

The inclusion of steady-state initialization in RELAP5 will require addition of several new subroutines, common blocks and modification of some of the existing coding. The changes required for steady-state initialization will be done in a phased approach, where upon completion of milestone, (i.e., single-phase fluid initialization, two-phase fluid initialization, heat conduction initialization, etc.) the changes will be saved as a new intermediate version. This approach will result in several sets of change card cards called "updates". These change cards will be maintained in a form so that when the project is complete, they can be easily reincorporated in the mainline version of RELAP5.

A utility program called SLIB77 will be used during the development to track all the changes made to the RELAP5 source (Ref. 5). SLIB77 is a computer program that is designed to maintain a source code and track all changes made to that source code. SLIB77 has all the features of the CDC maintenance program UPDATE that has traditionally be used to manage source code and changes on CDC mainframes. The advantage to using SLIB77 is that it is portable and works on a wide variety of computer platforms including PCs, workstations and mainframes. 
SLIB77 allows subroutines within a source to be identified uniquely with a DECK name. Each FORTRAN line within a DECK is assigned an identifier comprised of the DECK name and number sequence. The FORTRAN statement can then be modified, added, deleted or replaced by referring to the line identifier.

SLIB77 will be run a in batch mode in which a group of changes can be made on a temporary basis for debugging or on a permanent basis. If the changes are made permanently, SLIB77 has provisions to remove a change set at a later date and restore the original coding. As stated earlier, a set of change cards will be incorporated into the RELAP5 source permanently only as major portions of the steady-state initialization has been developed and debugged. These change sets will create intermediate working versions of the modified code. All sets of the change cards will be kept so at the completion of the project, the steps to create the final RELAP5 version can be recreated at another site and computer platform.

As shown in flow diagrams and discussed in the text, most all changes will be in the form of new subroutines or small updates to existing routines. These changes can be added using IF-THEN constructs that are activated using SLIB77's DEFINE capability. 


\section{DEMONSTRATION ANALYSIS}

Five steady-state demonstration analyses were performed with the RELAP5 testbed code In all cases, the models consisted of several consecutive volumes and junctions forming a channel bounded on each end by time-dependent volumes The initial fluid flow rate was input and the code was used to compute the pressure distribution down the channel The five demonstration test problems are

(1) FRIGG FT-6 Experiment with Zero Power

(2) Single Contraction

(3) Single Expansion

(4) Several Expansıons and Contractions

(5) Same as Case 1 With Power Addition

The demonstration problems were run with the testbed code using steady-state initialization and then rerun with standard RELAP5 using a transient solution to achieve steady-state conditions For the testbed code run, the pressures at both ends of the pipe are input (tıme-dependent volumes), the flow was input, and all loss coefficients were input except for the exit junction Steady-state Initialization was used to compute the volume pressure distribution within the channel and the one unknown loss coefficient To verify that the steady-state ınıtıalızation was performing properly, the same cases were run with standard RELAP5 using a transient solution For the standard RELAP5 runs, the same pressure boundaries were used, the unknown loss coefficient computed from the steady-state run was input and the flow through the channel and pressure distribution was computed The pressure distribution, phasic velocities and mass flow rates matched for the two methods in all cases, indicatıng the testbed code was functionıng correctly

\subsection{Test Problem 1}

A schematic for the Test Problem 1 model is shown in Figure 101 As shown, time-dependent volumes are used to provide pressure boundary conditions to a ten-cell annulus (pipe) component For this steady-state testbed demonstration analysis, all the normal volume pressures were input as the same value (182 $75 \mathrm{psia})$ and an initial mass flow rate of $948 \mathrm{lb} / \mathrm{sec}$ The input volume pressure was supplied as a flat pressure profile since all that is required is a guess at the final steady-state value The boundary volumes were initialized using the conditions shown in Figure 101 A pressure differential of 645 psi was applied using the time-dependent pressure boundaries The specified mass flow rate was propagated through the annulus and the loss coefficient in junction 99 was computed to balance the total pressure drop defined by the two boundary pressures In this example, the internal pressure distribution is computed The testbed code was then run to obtaın a steady state solution

The steady-state pressure distribution computed using the testbed code converged to 001 psi on the third iteration and the exit loss coefficient converged to $10^{8}$ on the fifth iteration Each of the steady-state iterations is computationally equivalent to a single tıme-step advancement in the transient solution scheme Table 101 shows the pressure distribution and junction 99 loss coefficient for the five iterations taken 


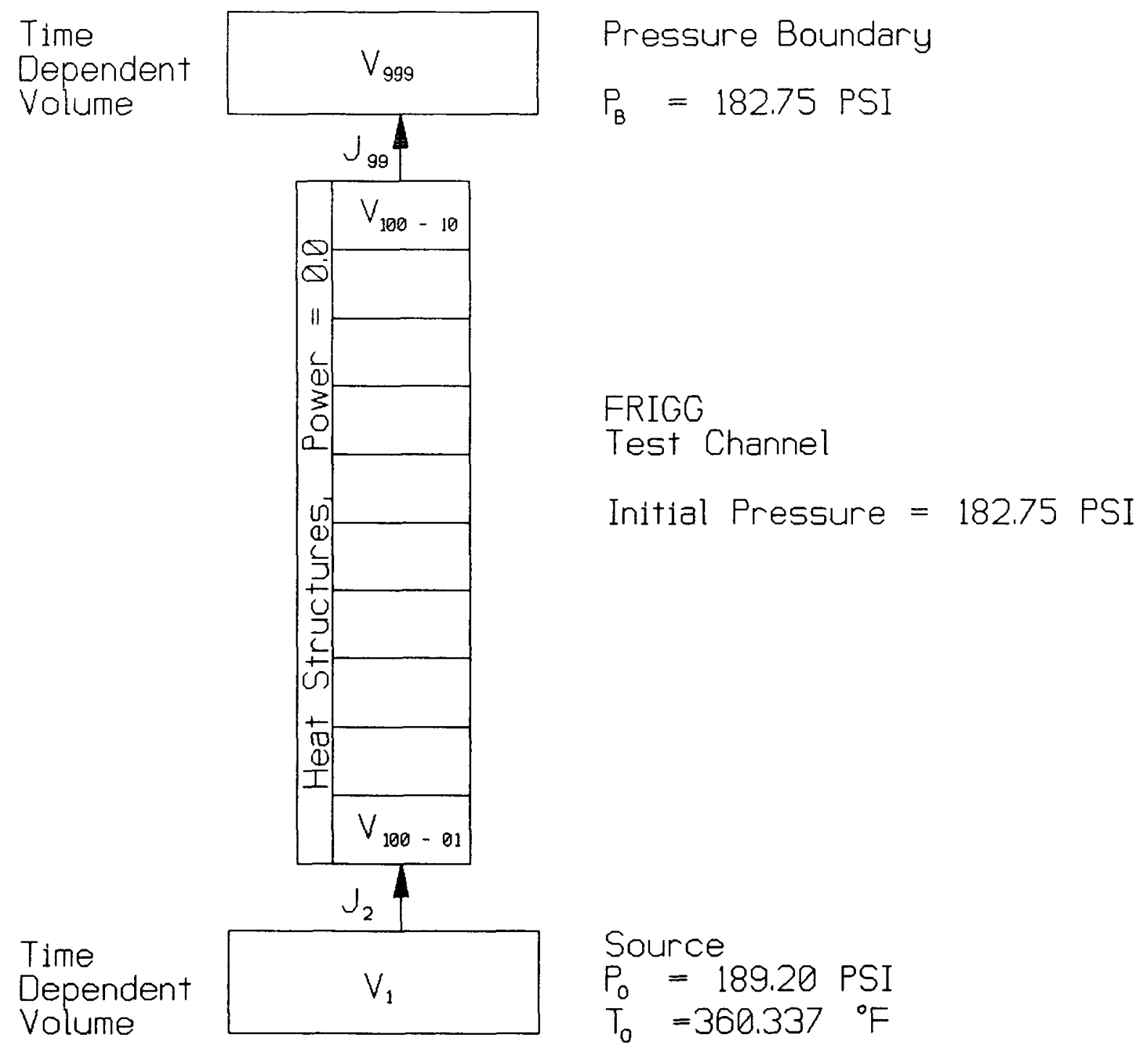

Figure 10.1 Noding diagram for FRIGG test FT-6 
Table 10.1 Pressure and loss coefficient convergence steady-state test problem

\begin{tabular}{|c|c|c|c|c|c|c|}
\hline \multirow[b]{2}{*}{ Node } & \multicolumn{6}{|c|}{ Pressure At Iteration } \\
\hline & 0 & 1 & 2 & 3 & 4 & 5 \\
\hline 1 & 189.20 & 189.20 & 189.20 & 189.20 & 189.20 & 189.20 \\
\hline $100-01$ & 182.75 & 188.57 & 188.73 & 188.73 & 188.73 & 188.73 \\
\hline $100-02$ & 182.75 & 187.96 & 188.12 & 188.12 & 188.12 & 188.12 \\
\hline $100-03$ & 182.75 & 187.32 & 187.48 & 187.48 & 187.48 & 187.48 \\
\hline $100-04$ & 182.75 & 186.71 & 186.87 & 186.87 & 186.87 & 186.87 \\
\hline $100-05$ & 182.75 & 186.07 & 186.23 & 186.23 & 186.23 & 186.23 \\
\hline $100-06$ & 182.75 & 185.46 & 185.62 & 185.62 & 185.62 & 185.62 \\
\hline $100-07$ & 182.75 & 184.81 & 184.98 & 184.98 & 184.98 & 184.98 \\
\hline $100-08$ & 182.75 & 184.20 & 184.37 & 184.37 & 184.37 & 184.37 \\
\hline $100-09$ & 182.75 & 183.56 & 183.72 & 183.72 & 183.72 & 183.72 \\
\hline $100-10$ & 182.75 & 182.95 & 183.11 & 183.11 & 183.11 & 183.11 \\
\hline 999 & 182.75 & 182.75 & 182.75 & 182.75 & 182.75 & 182.75 \\
\hline $\begin{array}{l}\text { Jun } 100-05 \mathrm{Vel} \text {. }(\mathrm{ft} / \mathrm{s}) \\
\text { Jun } 99 \mathrm{Vel} .(\mathrm{ft} / \mathrm{s})\end{array}$ & $\begin{array}{l}5.2318 \\
5.2319\end{array}$ & & & & & \\
\hline Loss Coefficient & 0.421 & 0.37835855 & 0.74702021 & 0.74705900 & 0.74706182 & 0.74706182 \\
\hline
\end{tabular}

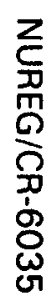


The RELAP5 transient solution was then used to obtain the steady-state solution for the same problem. The initial condition specifications were identical to those used with the testbed code except that the junctions were initialized to the phasic velocities computed by the testbed code and the loss coefficient computed at junction 99 was input with eight-digit accuracy.

A steady-state solution identical to the one obtained with the testbed code was obtained after nine seconds of transient simulation. As shown in Table 10.2, the pressure reached the steady-state values (five significant figures) in five seconds (68 time steps) and the velocities reached steady state at nine seconds (108 time steps).

\subsection{Test Problems 2, 3, and 4}

To more completely test the initial testbed steady-state code modifications, three additional problems were executed. The problems were run primarily to test momentum flux terms and further verify the loss coefficient computation. Problems 2 and 3 tested a single contraction and expansion, respectively, and Problem 4 tested a combination of expansions and contractions as shown in Figure 10.2 and was vertically oriented. For each problem, the steady-state testbed code was first executed. Then the computed loss coefficient was input to the RELAP5 transient code, which was then run to a steady state. For all three problems identical steady states were obtained using both methods. Initial conditions and converged steady-state values are tabulated in Tables 10.3 and 10.4 .

\subsection{Test Problem 5}

Test Problem 5 is the same configuration as Test Problem 1 except it was executed with the power for the FRIGG experiment at $10 \%$ power. This allowed the problem to utilize the steady-state heat transfer conduction calculation described in Section 4, but still remain subcooled. With no thermal time constants, the steady-state conduction is converged each iteration, using only one or two conduction solution iterations. The problem is hydraulically and thermally converged at steady state in five iterations.

RELAP5 was executed using the loss coefficient computed from the steady-state testbed code. Thermal and hydraulic convergence was obtained between nine to ten seconds, or approximately 100-110 time advancements. The RELAP5 and steady-state testbed code obtained identical results. 
Table 10.2 RELAP5 pressure and velocity solution

\begin{tabular}{|c|c|c|c|c|}
\hline \multirow[b]{2}{*}{ Time (s) } & \multicolumn{2}{|c|}{ Pressure (psi) } & \multicolumn{2}{|c|}{ Velocity $(\mathrm{ft} / \mathrm{s})$} \\
\hline & Node 100-05 & Node $100-10$ & Jun 100-05 & Jun 99 \\
\hline 0.0 & 182.75 & 182.75 & 5.2319 & 5.2319 \\
\hline 0.1 & 210.23 & 185.28 & 28.777 & 28.772 \\
\hline 0.2 & 122.33 & 179.09 & -30.087 & -30.073 \\
\hline 0.3 & 188.03 & 179.51 & -23.011 & -23.015 \\
\hline 0.4 & 184.41 & 180.97 & -17.392 & -17.395 \\
\hline 0.5 & 185.13 & 181.69 & -13.716 & -13.716 \\
\hline 0.6 & 185.22 & 182.17 & -11.138 & -11.139 \\
\hline 0.7 & 185.77 & 182.46 & -9.2116 & -9.2120 \\
\hline 0.8 & 185.93 & 182.65 & -7.7017 & -7.7020 \\
\hline 0.9 & 186.03 & 182.77 & -6.4736 & -6.4738 \\
\hline 1.0 & 186.11 & 182.86 & -5.4438 & -5.4440 \\
\hline 1.5 & 186.28 & 183.04 & -1.8505 & -1.8506 \\
\hline 2.0 & 186.30 & 183.07 & 0.78999 & 0.78999 \\
\hline 2.5 & 186.27 & 183.08 & 2.9417 & 2.9417 \\
\hline 3.0 & 186.25 & 183.10 & 4.2057 & 4.2058 \\
\hline 3.5 & 186.24 & 183.11 & 4.8044 & 4.8045 \\
\hline 4.0 & 186.23 & 183.11 & 5.0597 & 5.0598 \\
\hline 5.0 & 186.23 & 183.11 & 5.2051 & 5.2052 \\
\hline 6.0 & 186.23 & 183.11 & 5.2279 & 5.2280 \\
\hline 7.0 & 186.23 & 183.11 & 5.2313 & 5.2314 \\
\hline 8.0 & 186.23 & 183.11 & 5.2318 & 5.2319 \\
\hline \multirow[t]{2}{*}{9.0} & 186.23 & 183.11 & 5.2318 & 5.2319 \\
\hline & \multicolumn{4}{|c|}{ Converged at 9 seconds (108 time steps) } \\
\hline
\end{tabular}




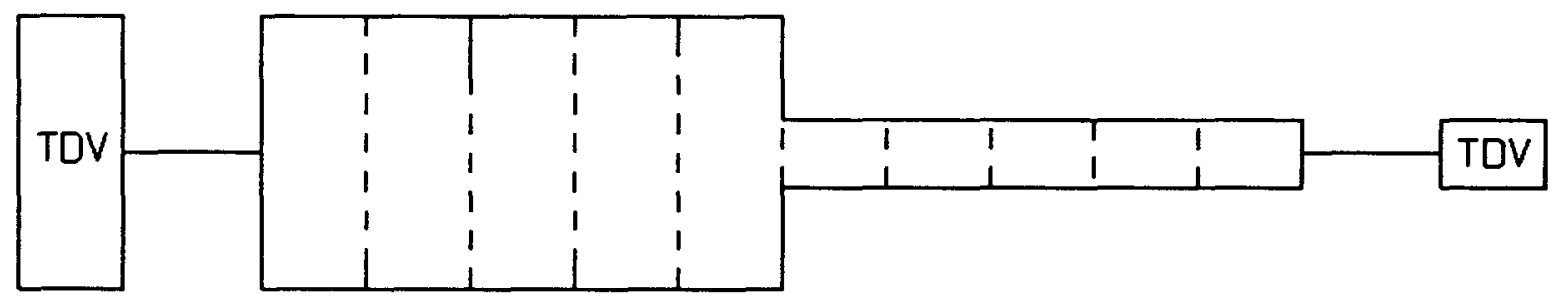

Problem 2 - Contraction

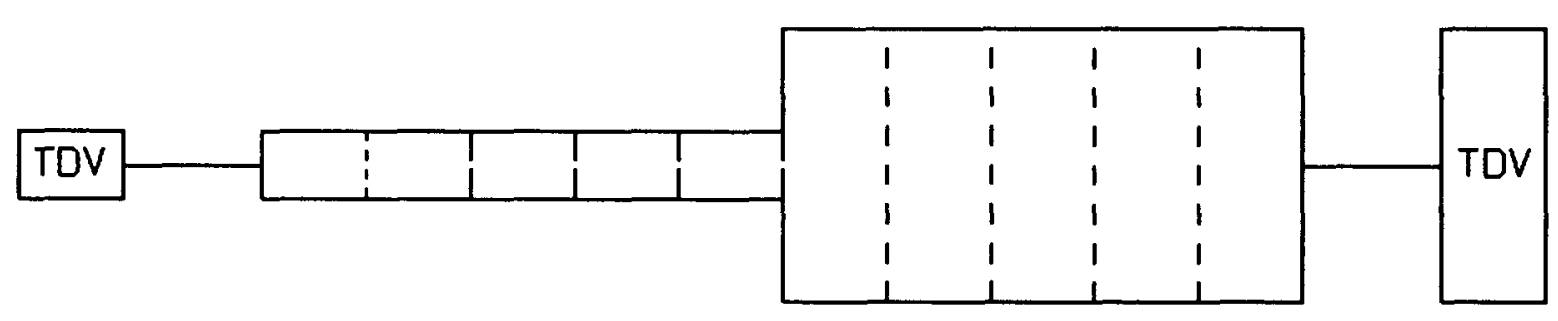

Problem 3 - Expansion

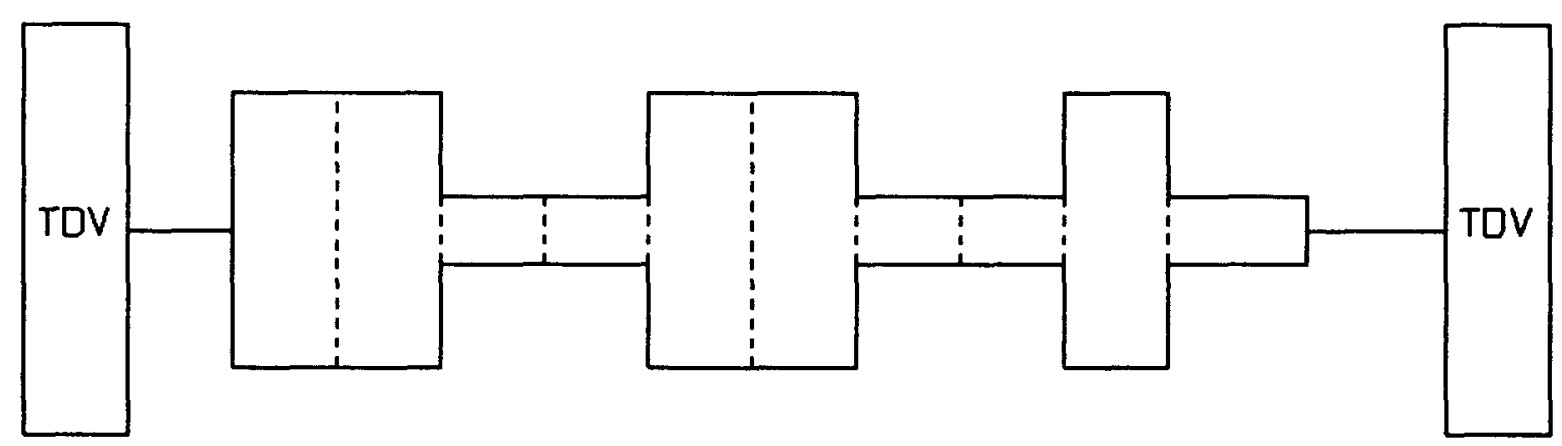

Problem 4 - Contractions and Expansions

Figure 10.2 Schematic diagrams for test problems 2, 3, and 4 
Table 10.3 Data for test problems 2, 3, and 4

\begin{tabular}{llcc}
\hline & PROB2 & PROB3 & PROB4 \\
\hline Inlet to Volume Pressure (psi) & 195.00 & 195.00 & 193.00 \\
Annulus Initial Pressure (psi) & 182.75 & 182.75 & 182.75 \\
Outlet TD Volume Pressure (psi) & 182.75 & 182.75 & 182.75 \\
Inlet TD Volume Flow Area $\left(\mathrm{ft}^{2}\right.$ ) & 0.032816 & 0.0100 & 100.00 \\
Restricted Annulus Flow Area $\left(\mathrm{ft}^{2}\right)$ & 0.0100 & 0.0100 & 0.0100 \\
Expanded Annulus Flow Area $\left(\mathrm{ft}^{2}\right.$ ) & 0.032816 & 0.032816 & 0.032816 \\
Outlet TD Volume Flow Area $\left(\mathrm{ft}^{2}\right.$ ) & 0.0100 & 0.032816 & 0.041424 \\
Annulus Node Length $(\mathrm{ft})$ & 1.45013 & 1.45013 & 1.45013 \\
Number of Nodes & 10 & 10 & 10 \\
Orientation & Horizontal & Horizontal & Vertical \\
Input Flow Rate (lbm/s) & 9.480 & 9.480 & 9.480 \\
\hline
\end{tabular}


Table 10.4 Calculation results for problems 2,3 , and 4

\begin{tabular}{lccc}
\hline & PROB2 & PROB3 & PROB4 \\
\hline Computed Loss Coefficient & 4.09206 & 63.2927 & 0.298527 \\
Mass Flow Rate (lbm/s) & & & \\
& 9.480 & 9.480 & 9.480 \\
Velocity in Contraction (ft/s) & & & \\
& 17.168 & 17.168 & 17.169 \\
Velocity in Expansion (ft/s) & & & \\
Annulus Pressure (psi) & 5.2316 & 5.2317 & \\
V100-01 & & & \\
V100-02 & 194.97 & 194.2318 \\
V100-03 & 194.92 & 194.26 & 191.92 \\
V100-04 & 194.83 & 193.44 & 189.17 \\
V100-05 & 194.78 & 192.95 & 188.12 \\
V100-06 & 194.69 & 192.12 & 188.55 \\
V100-07 & 192.83 & 193.44 & 187.94 \\
V100-08 & 192.00 & 193.36 & 184.13 \\
V100-09 & 191.51 & 193.30 & 184.56 \\
V100-10 & 190.69 & 193.22 & 182.14 \\
Steady-State Iterations & 190.19 & 193.16 & 2 \\
Transient Time Steps & & & 30 \\
\hline & 3 & 4 & 7 \\
\hline
\end{tabular}




\section{REFERENCES}

1. K. E. Carlson, et al., "RELAP5/MOD3 Code Manual - Volume 1: Code Structure, System Models, and Solution Methods (Draft)", NUREG/CR-5535, June 1990.

2. J. H. McFadden, et al., "RETRAN-02 - A Program for Transient Thermal-Hydraulic Analysis of Complex Fluid Flow Systems: Volume 1 - Equations and Numerics", EPRI CCM-1850, April 1981, revised June 1987.

3. J. H. McFadden, et al., "RETRAN-03 - A Program for Transient Thermal-Hydraulic Analysis of Complex Fluid Flow Systems: Volume 1 - Computer Code Manual", EPRI CCM-7450, May 1992.

4. C. E. Peterson and M. P. Paulsen, "A RETRAN-03 Steady-State Initialization Method for Systems at Low Power", Proceedings of the Fifth International Topical Meeting on Reactor Thermal Hydraulics (NURETH-5), Salt Lake City, September 1992.

5. A. Lunsford, "SLIB77 - Reference Manual", Western Area Power Administration, January 1991. 